\title{
Exhausting all exact solutions of BPS domain wall networks in arbitrary dimensions
}

\author{
Minoru Eto $\odot,{ }^{1,2, *}$ Masaki Kawaguchi $\odot,{ }^{1, \dagger}$ Muneto Nitta, ${ }^{2,3, *}$ and Ryotaro Sasaki ${ }^{1}$ \\ ${ }^{1}$ Department of Physics, Yamagata University, Kojirakawa-machi 1-4-12, \\ Yamagata, Yamagata 990-8560, Japan \\ ${ }^{2}$ Research and Education Center for Natural Sciences, Keio University, Hiyoshi 4-1-1, \\ Yokohama, Kanagawa 223-8521, Japan \\ ${ }^{3}$ Department of Physics, Keio University, Hiyoshi 4-1-1, \\ Yokohama, Kanagawa 223-8521, Japan
}

(Received 15 April 2020; accepted 15 May 2020; published 28 May 2020)

\begin{abstract}
We obtain full moduli parameters for generic nonplanar Bogomol'nyi-Prasad-Sommerfield networks of domain walls in an extended Abelian-Higgs model with $N$ complex scalar fields and exhaust all exact solutions in the corresponding $\mathbb{C} P^{N-1}$ model. We develop a convenient description by grid diagrams which are polytopes determined by mass parameters of the model. To illustrate the validity of our method, we work out nonplanar domain wall networks for lower $N$ in $3+1$ dimensions. In general, the networks can have compact vacuum bubbles, which are finite vacuum regions surrounded by domain walls, when the polytopes of the grid diagrams have inner vertices, and the size of bubbles can be controlled by moduli parameters. We also construct domain wall networks with bubbles in the shapes of the Platonic, Archimedean, Catalan, and Kepler-Poinsot solids.
\end{abstract}

DOI: 10.1103/PhysRevD.101.105020

\section{INTRODUCTION}

It sometimes happens that systems have multiple discrete vacua or ground states, which is inevitable when a discrete symmetry is spontaneously broken. In such a case, there appear domain walls (or kinks) in general [1-3] which are inevitably created during second order phase transitions [4-7]. They are the simplest topological solitons appearing in various condensed matter systems such as magnets [8], graphenes [9], carbon nanotubes, superconductors [10], atomic Bose-Einstein condensates [11], and helium superfluids $[6,7,12]$, as well as high density nuclear matter $[13,14]$, quark matter [15], and early Universe [4,16]. In cosmology, cosmological domain wall networks are suggested as a candidate of dark matter and/or dark energy [17].

As the cases of other topological solitons, domain walls can become Bogomol'nyi-Prasad-Sommerfield (BPS) states $[18,19]$, attaining the minimum energy for a fixed boundary condition and satisfy first order differential

\footnotetext{
*meto@sci.kj.yamagata-u.ac.jp †ddwbb.daigaku@gmail.com

\#nitta@phys-h.keio.ac.jp
}

Published by the American Physical Society under the terms of the Creative Commons Attribution 4.0 International license. Further distribution of this work must maintain attribution to the author(s) and the published article's title, journal citation, and DOI. Funded by SCOAP. equations called BPS equations. In such cases, one can often embed the theories to supersymmetric (SUSY) theories by appropriately adding fermion superpartners, in which BPS solitons preserve some fractions of SUSY. Their topological charges are central (or tensorial) charges of corresponding SUSY algebras. The BPS domain walls in $3+1$ dimensions were studied extensively in field theories with both $\mathcal{N}=1$ SUSY [20-35] and $\mathcal{N}=2$ SUSY [36-56]; see Refs. [57-60] as a review. They preserve a half of SUSY and thereby are called $\frac{1}{2}$ BPS states, accompanied by SUSY central (tensorial) charges $Z_{m}\left(m=1,2\right.$; labeling spatial coordinates $x^{m}$ perpendicular to the domain wall) as domain wall topological charges $[22,24,61]$. In general, if several domain walls meet along a line, it forms a planar domain wall junction. In SUSY models, the planar domain wall junctions preserve a quarter SUSY [62-64], therefore are called $\frac{1}{4}$ BPS states, accompanied by a junction topological charge $Y$ in addition to $Z_{m}(m=1,2)$. The $\frac{1}{4}$ BPS domain wall junctions have been studied in theories with $\mathcal{N}=1$ SUSY [32,65-73] and $\mathcal{N}=2$ SUSY [74-83]. In the $\mathcal{N}=2$ SUSY gauge models, not only planar domain wall junctions, but also planar domain wall networks as $\frac{1}{4}$ BPS states were constructed $[75,76]$. The low-energy effective action for normalizable modes within the networks was obtained [78] and applied to study of low-energy dynamics [79]. Non-BPS planer domain wall networks were also studied in Refs. [84,85]. 
Recently, the present authors proposed a model, a $D+1$ dimensional $U(1)$ gauge theory [86] admitting novel analytic solutions of the BPS single nonplanar domain wall junctions. This model cannot be made supersymmetric but still admits stable BPS states so that we can use the well-known Bogomol'nyi completion technique to derive BPS equations. The model consists of $N$ charged complex scalar fields and $N^{\prime}$ neutral scalar fields coupled to the $U(1)$ gauge field. In Ref. [86], we restricted ourself to the special numbers $N-1=N^{\prime}=D$ and imposed the invariance under the symmetric group $\mathcal{S}_{D+1}$ of the rank $D+1$, which are the symmetry groups of the regular $D$ simplex.

In this paper, we investigate generic nonplanar networks of BPS domain walls in $D+1$ dimensions. We consider the generic case of $N \geq D+1$ imposing no discrete symmetry and exhaust all exact solutions with full moduli of generic BPS nonplanar networks of domain walls in the infinite $U(1)$ gauge coupling limit in which the model reduces to the $\mathbb{C} P^{N-1}$ model. These are the first exact solutions of nonplanar domain wall networks in $D$ dimensions $(D \geq 3)$. We first derive the BPS equations for generic Abelian gauge theories in $D+1$ dimensions. Then, we partially solve them by the moduli matrix formalism [58] and find all moduli parameters of the generic domain wall network solutions. We then demonstrate several concrete nonplanar networks in the $\mathbb{C} P^{N-1}$ model in $D=3$ for $N=4,5,6$. In the case of $N=4$, the solution has only one junction at which four vacua meet. Network structures appear for $N>4$. In the case of $N=5$, we show two different types of networks exist in general. The first type has a vacuum bubble (a compact vacuum domain) surrounded by semiinfinite vacuum domains. Instead, the second type does not have any bubbles but all the vacuum domains are semiinfinitely extended. In the $N=6$ case, there are three different types according to the number of the vacuum bubbles, two, one, or zero. Finally, we find a connection to the well-known polyhedra known from ancient times. Indeed, we find the vacuum bubbles which are congruent with five Platonic solids. In addition, the Archimedean and the Catalan solids appear as the vacuum bubbles. We also construct the Kepler-Poinsot star solids as domain wall networks.

This paper is organized as follows. In Sec. II, we introduce our model. In Sec. III, we derive the BPS equations and clarify the moduli space of the BPS solutions. In Sec. IV, we first consider the infinite $U(1)$ gauge coupling limit in which the model reduces to the massive $\mathbb{C} P^{N-1}$ nonlinear sigma model. We then exhaust all exact solutions with full moduli parameters for the BPS equations. We further give several examples of nonplanar networks in $D=3$. In Sec. V, we study relations between the domain wall networks in $D=3$ and the classic solids, like the Platonic, Archimedean, Catalan, and KeplerPoinsot solids. Finally, we summarize our results and give a discussion in Sec. VI.

\section{THE MODEL}

We study a $U(1)$ gauge theory with $N$ charged complex scalar fields $H^{A}(A=1,2, \ldots, N)$ and $N^{\prime}$ real scalar fields $\Sigma^{A^{\prime}}\left(A^{\prime}=1,2, \ldots, N^{\prime}\right)$ in $D+1$-dimensional spacetime. The Lagrangian is given by

$$
\begin{aligned}
\mathcal{L}= & -\frac{1}{4 e^{2}} F_{\mu \nu} F^{\mu \nu}+\frac{1}{2 e^{2}} \sum_{A^{\prime}=1}^{N^{\prime}} \partial_{\mu} \Sigma^{A^{\prime}} \partial^{\mu} \Sigma^{A^{\prime}} \\
& +D_{\mu} H\left(D^{\mu} H\right)^{\dagger}-V \\
V=\frac{1}{2 e^{2}} Y^{2} & +\sum_{A^{\prime}=1}^{N^{\prime}}\left(\Sigma^{A^{\prime}} H-H M^{A^{\prime}}\right)\left(\Sigma^{A^{\prime}} H-H M^{A^{\prime}}\right)^{\dagger},
\end{aligned}
$$

where $H$ is an $N$ component row vector made of $H^{A}$,

$$
H=\left(H^{1}, H^{2}, \ldots, H^{N}\right),
$$

$Y$ is a scalar quantity defined by

$$
Y=e^{2}\left(v^{2}-H H^{\dagger}\right),
$$

and $M^{A^{\prime}}\left(A^{\prime}=1, \ldots, N^{\prime}\right)$ are $N$ by $N$ real diagonal mass matrices defined by

$$
M^{A^{\prime}}=\operatorname{diag}\left(m_{A^{\prime}, 1}, m_{A^{\prime}, 2}, \ldots, m_{A^{\prime}, N}\right) .
$$

The spacetime index $\mu$ runs from 0 to $D$, and $F_{\mu \nu}$ is an $U(1)$ gauge field strength. The coupling constants in the Lagrangian in Eq. (2.1) are taken to be the so-called Bogomol'nyi limit. For later use, let us define $\boldsymbol{m}_{A}$ by an $N^{\prime}$ vector whose components are the Ath diagonal elements of $M^{A^{\prime}}$ s, namely,

$$
\boldsymbol{m}_{A}=\left(m_{1, A}, m_{2, A}, \ldots, m_{N^{\prime}, A}\right) .
$$

In the following, we will mostly consider generic masses:

$$
\boldsymbol{m}_{A} \neq \boldsymbol{m}_{B}, \quad \text { if } A \neq B .
$$

Since the scalar potential $V$ is positive semidefinite, a classical vacuum of the theory is determined by $V=0$ : $H H^{\dagger}=v^{2}$, and $\Sigma^{A^{\prime}} H-H M^{A^{\prime}}=0$. In the generic case of Eq. (2.7), there are $N$ discrete vacua. The $A$ th vacua which we will denote by $\langle A\rangle$ is given by $H^{B}=v \delta_{A}^{B}$, and $\Sigma^{B^{\prime}}=m_{B^{\prime}, A}$. Simply, the vacua can be identified to the discrete points determined by the mass vectors $\left\{\boldsymbol{m}_{A}\right\}$ in the $\Sigma$ space,

$$
\langle A\rangle: \boldsymbol{\Sigma}=\boldsymbol{m}_{A} .
$$

The Lagrangian (2.1) is motivated by supersymmetry. In fact, when $N_{\mathrm{F}}^{\prime}=2$, it is a bosonic part of an $\mathcal{N}=2$ 
supersymmetric theory with eight supercharges in four dimensions.

\section{SOLVING BPS EQUATIONS FOR DOMAIN WALL NETWORKS}

\section{A. Derivation of the BPS equations}

From now on, we investigate BPS states of $\mathcal{L}$ in Eq. (2.1) in the case that the number $N^{\prime}$ of flavors for real adjoint scalars is equal to the number of the spatial dimensions $D .{ }^{1}$ In what follows, the Roman index $m$ stands not only for the spacial index as $m=1,2, \ldots, D$ but also for the index of $N^{\prime}$ $\left(m \equiv A^{\prime}\right)$. Then, the standard Bogomol'nyi completion for this system goes as follows:

$$
\begin{aligned}
\mathcal{E}= & \frac{1}{2 e^{2}} \sum_{m>n}\left\{F_{m n}^{2}+\left(\partial_{m} \Sigma_{n}-\xi_{m} \xi_{n} \partial_{n} \Sigma_{m}\right)^{2}\right\} \\
& +\frac{1}{2 e^{2}}\left(\sum_{m} \xi_{m} \partial_{m} \Sigma_{m}-Y\right)^{2} \\
& +\sum_{m}\left\{D_{m} H+\xi_{m}\left(\Sigma_{m} H-H M_{m}\right)\right\} \\
& \times\left\{D_{m} H+\xi_{m}\left(\Sigma_{m} H-H M_{m}\right)\right\}^{\dagger} \\
& +\sum_{m} \xi_{m} \mathcal{Z}_{m}+\sum_{m>n} \xi_{m} \xi_{n} \mathcal{Y}_{m n}+\sum_{m} \partial_{m} \mathcal{J}_{m},
\end{aligned}
$$

with $\xi_{m}= \pm 1$, and we have defined the domain wall topological charge density $\mathcal{Z}_{m}$, the domain wall junction charge density $\mathcal{Y}_{m n}$, and $\mathcal{J}_{m}$ by

$$
\begin{gathered}
\mathcal{Z}_{m}=v^{2} \partial_{m} \Sigma_{m} \\
\mathcal{Y}_{m n}=-\frac{1}{e^{2}} \operatorname{det}\left(\begin{array}{cc}
\partial_{m} \Sigma_{m} & \partial_{m} \Sigma_{n} \\
\partial_{n} \Sigma_{m} & \partial_{n} \Sigma_{n}
\end{array}\right), \\
\mathcal{J}_{m}=-\xi_{m}\left(\Sigma_{m} H-H M_{m}\right) H^{\dagger}
\end{gathered}
$$

respectively. The contribution by $\mathcal{J}_{m}$ vanishes under the space integrations since it is asymptotically zero because of the vacuum condition $\Sigma_{m} H-H M_{m}=0$.

The domain wall tension $Z_{m}$ measured along the $x^{m}$ direction can be defined from $\mathcal{Z}_{m}$ by

$Z_{m}=\int_{-\infty}^{\infty} d x^{m} \xi_{m} \mathcal{Z}_{m}=v^{2} \xi_{m}\left(\left.\Sigma_{m}\right|_{x^{m}=+\infty}-\left.\Sigma_{m}\right|_{x^{m}=-\infty}\right) \geq 0$

(no sum over $m$ ).

Note that $Z_{m}$ is always positive regardless of the choice of $\xi_{m}$. Hence, the genuine tension measured along the normal

\footnotetext{
${ }^{1}$ In Ref. [86], we considered the same model with restricted to a special case in which the flavor number $N$ is also related to the spatial dimensions, $N=D+1$. Instead, this work studies the case with generic $N(\geq D+1)$.
}

direction to the domain wall interpolating the vacua $\langle A\rangle$ and $\langle B\rangle$ is given by

$$
|\boldsymbol{Z}|=v^{2}\left|\boldsymbol{m}_{A}-\boldsymbol{m}_{B}\right|
$$

On the other hand, the domain wall junction charge can be defined from the topological charge density $\mathcal{Y}_{m n}$ as

$$
Y_{m n}=\xi_{m} \xi_{n} \int d x^{m} d x^{n} \mathcal{Y}_{m n}=-\frac{\xi_{m} \xi_{n}}{e^{2}} S_{m n} \leq 0,
$$

negatively contributing to the BPS energy. Here, we have defined

$$
S_{m n} \equiv \int d x^{m} d x^{n} \operatorname{det}\left(\begin{array}{ll}
\partial_{m} \Sigma_{m} & \partial_{m} \Sigma_{n} \\
\partial_{n} \Sigma_{m} & \partial_{n} \Sigma_{n}
\end{array}\right) .
$$

The integrand is a Jacobian of a map from the whole $x^{m}-x^{n}$ plane to a region in the $\Sigma^{m}-\Sigma^{n}$ plane defined by the function $\left(\Sigma_{m}\left(x^{m}, x^{n}\right), \Sigma_{n}\left(x^{m}, x^{n}\right)\right)$ with all other coordinates $x^{k}$ $(k \neq m, n)$ fixed. $S_{m n}$ can be either positive or negative, and its absolute value is the area of the image. Nevertheless, $Y_{m n}$ is always negative since $S_{m n}$ is accompanied with $\left(-\xi_{m} \xi_{n}\right)$, and so it should be understood as a sort of binding energy among domain walls [67-69].

Since the first three terms of Eq. (3.1) are positive semidefinite, the Bogomol'nyi energy bound is given by

$$
\mathcal{E} \geq \sum_{m} \xi_{m} \mathcal{Z}_{m}+\sum_{m>n} \xi_{m} \xi_{n} \mathcal{Y}_{m n}+\sum_{m} \partial_{m} \mathcal{J}_{m}
$$

and it is saturated by the BPS states satisfying the BPS equations,

$$
\begin{gathered}
F_{m n}=0, \\
\xi_{n} \partial_{m} \Sigma_{n}-\xi_{m} \partial_{n} \Sigma_{m}=0, \\
\xi_{m} D_{m} H+\Sigma_{m} H-H M_{m}=0, \\
\sum_{m} \xi_{m} \partial_{m} \Sigma_{m}-Y=0,
\end{gathered}
$$

where $m, n=1,2, \ldots, D$. One can verify that all solutions of the above BPS equations solve the full equations of motion. This is the $D$-dimensional extension of the $\frac{1}{4}$ BPS equations of the planar domain wall junction in $D=2$ cases studied in Refs. [74,75,78,79].

In the previous work [86] by the present authors, we generalized the topological charge densities $\mathcal{Z}_{m}$ and $\mathcal{Y}_{m n}$ under the observation that $\mathcal{Z}_{m}$ and $\mathcal{Y}_{m n}$ are nothing but oneand two-dimensional Jacobians of maps $x^{m} \rightarrow \Sigma_{m}$ and $\left(x^{m}, x^{n}\right) \rightarrow\left(\Sigma_{m}, \Sigma_{n}\right)$, respectively. For a domain wall interpolating the $\langle A\rangle$ and $\langle B\rangle$ vacua, an integral of $\mathcal{Z}_{m}$ (with an appropriate rescale to make it dimensionless) 
measures a covering number of a map from $\mathbb{R}^{1}(-\infty<$ $\left.x^{m}<\infty\right)$ onto the one-dimensional interval $\left(m_{m, A}<\Sigma_{m}<\right.$ $\left.m_{m, B}\right)$. The charge is topological and indeed takes values either $+1,0$, or -1 . Similar arguments hold for $\mathcal{Y}_{m n}$, and it is straightforward to generalize it in $D$ dimensions as

$$
\begin{aligned}
& \mathcal{W}_{d}\left(m_{1}, m_{2}, \ldots, m_{d}\right) \\
& =\operatorname{det}\left(\begin{array}{cccc}
\partial_{m_{1}} \Sigma_{m_{1}} & \partial_{m_{1}} \Sigma_{m_{2}} & \cdots & \partial_{m_{1}} \Sigma_{m_{d}} \\
\partial_{m_{2}} \Sigma_{m_{1}} & \partial_{m_{2}} \Sigma_{m_{2}} & \cdots & \partial_{m_{2}} \Sigma_{m_{d}} \\
\vdots & & \ddots & \vdots \\
\partial_{m_{d}} \Sigma_{m_{1}} & \partial_{m_{d}} \Sigma_{m_{2}} & \cdots & \partial_{m_{d}} \Sigma_{m_{d}}
\end{array}\right),
\end{aligned}
$$

where $1 \leq d \leq D, \quad m_{\alpha} \in\{1,2, \ldots, D\} \quad(\alpha=1,2, \ldots, d)$, and $m_{\alpha}>m_{\beta}$ if $\alpha>\beta$. We have $\mathcal{W}_{1}(m) \propto \mathcal{Z}_{m}$ and $\mathcal{W}_{2}(m, n) \propto \mathcal{Y}_{m n}$. In general, the BPS solutions in $D$ dimensions have $d$-dimensional substructures, and $\mathcal{W}_{d}$ provides us topological numbers associated with the substructures.

\section{B. The moduli matrix method}

Let us solve the BPS equations (3.10)-(3.13). To this end, we first introduce a complex scalar function $S\left(x^{m}\right)$ by

$$
A_{m}-i \xi_{m} \Sigma_{m}=-i \partial_{m} \log S .
$$

Then, Eqs. (3.10) and (3.11) are automatically satisfied. Plugging this into Eq. (3.12), we have

$$
\partial_{m} H+\left(\partial_{m} \log S\right) H-\xi_{m} H M_{m}=0 .
$$

This can be solved by

$$
H=v S^{-1} H_{0} e^{\xi_{m} M_{m} x^{m}},
$$

where $H_{0}$ is an arbitrary complex constant $N$ vector. $H_{0}$ is called the moduli matrix because the elements of $H_{0}$ are moduli (integration constants) of the BPS solutions. Finally, we are left with the fourth equation (3.13). To solve this, let us express the neutral real scalar fields $\Sigma_{m}$ in terms of $S$,

$$
\Sigma_{m}=\frac{1}{2} \xi_{m} \partial_{m} \log \Omega, \quad \Omega \equiv|S|^{2},
$$

where $\Omega$ is the gauge-invariant quantity. Using this, the fourth BPS equation (3.13) is cast into the following Poisson equation in $D$ dimensions:

$$
\frac{1}{2} \nabla^{2} \log \Omega=e^{2} v^{2}\left(1-\Omega^{-1} H_{0} e^{2 \xi_{m} M_{m} x^{m}} H_{0}^{\dagger}\right) .
$$

We call this the master equation for the BPS states.

We note that the original fields $A_{m}, \Sigma_{m}$, and $H$ are intact under the following transformation:

$$
\left(S, H_{0}\right) \rightarrow V\left(S, H_{0}\right), \quad V \in \mathbb{C}^{*},
$$

where $V$ is constant. This is called the $V$ transformation under which any physical information is independent.

To solve the master equation, we first need to fix the moduli matrix $H_{0}$. Once $H_{0}$ is given, the boundary condition at $|\boldsymbol{x}| \rightarrow \infty$ is automatically specified. Namely, we should solve the master equation with the boundary condition

$$
\lim _{|x| \rightarrow \infty} \Omega=H_{0} e^{2 \xi_{m} M_{m} x^{m}} H_{0}^{\dagger} .
$$

As a trivial example, let us consider a homogeneous vacuum, say the first vacuum $\langle 1\rangle$. The $\langle 1\rangle$ vacuum configuration is given by the moduli matrix

$$
H_{0}=\left(h_{1}, 0, \ldots, 0\right), \quad h_{1} \in \mathbb{C}^{*} .
$$

The corresponding master equation becomes

$$
\frac{1}{2} \nabla^{2} \log \Omega=e^{2} v^{2}\left(1-\Omega^{-1}\left|h_{1}\right|^{2} e^{2 \xi_{m} m_{m, 1} x^{m}}\right),
$$

which can be solved by

$$
\Omega=\left|h_{1}\right|^{2} e^{2 \xi_{m} m_{m, 1} x^{m}} .
$$

Plugging this into Eq. (3.18), we immediately find $\boldsymbol{\Sigma}=\boldsymbol{m}_{1}$. It is also straightforward to verify $H=(v, 0, \ldots, 0)$. Note that the constant $h_{1}$ in the moduli matrix does not play any role in the above example. This redundancy comes from the $V$ transformation in Eq. (3.20). Indeed, we could, from the first point, fix the moduli matrix (3.22) as $H_{0}=\left(h_{1}, 0, \ldots, 0\right) \rightarrow(1,0, \ldots, 0)$.

Nontrivial inhomogeneous solutions including a single domain wall connecting the $\langle A\rangle$ and $\langle B\rangle$ vacua are generated by the moduli matrix with the nonzero constants only in the Ath and $B$ th entries as

$$
\begin{aligned}
H_{0} & =\left(0, \ldots, 0,1,0, \ldots, 0, h_{B}, 0, \ldots, 0\right) \\
& \sim\left(0, \ldots, 0, h_{A}, 0, \ldots, 0,1,0, \ldots, 0\right) .
\end{aligned}
$$

The nonzero moduli parameters $h_{A}$ and $h_{B}$ are related by the $V$ transformation as $h_{A} h_{B}=1$.

Similarly, if we have $H_{0}$ with three nonzero constants, we will have a domain wall junction dividing the corresponding three vacua. The moduli matrix is given by

$H_{0}=\left(0, \ldots, 0,1,0, \ldots, 0, h_{B}, 0, \ldots, 0, h_{C}, 0, \ldots, 0\right)$.

Maximally complex solutions dividing $N$ vacuum domains in $D$ dimensions are, then, obtained by the moduli matrix which has no zeros in any elements. Such complicated extended objects in $D$ dimensions are not easy for us to handle without exact solutions. Unfortunately, the master 
equation (3.19) does not seem analytically solvable, except for the very special cases possessing the highest discrete symmetry group $\mathcal{S}_{D+1}$, the symmetric group of the degree $D+1$, in the model with the special number of the flavor $N=D+1$ and finely tuned parameters $g, c$, and $m_{A^{\prime}, A}$, as demonstrated in Ref. [86]. Even in the fine-tuned models, the exact analytic solution were only found for a single junction of the domain walls since $N=D+1$ is the minimum number. However, as we show in the next section, the solutions include domain wall networks when $N>D+1$.

To see shapes of domain wall networks, let us define a weight for each vacuum by

$$
w^{\langle A\rangle}=e^{\tilde{\boldsymbol{m}}_{A} \cdot \boldsymbol{x}+a_{A}}, \quad(A=1, \ldots, N) .
$$

Since the weight is the exponential function of the spatial coordinate, only one weight dominates the rest $N-1$ weights at each point $\boldsymbol{x}$. Suppose the weight of the $\langle A\rangle$ vacuum is dominant in vicinity of a point $\boldsymbol{x}_{0}$. There, we have $\Omega \sim\left(w^{\langle A\rangle}\right)^{2}$, and then $\boldsymbol{\Sigma}$ reads from Eq. (3.18),

$$
\left.\boldsymbol{\Sigma}\right|_{x \sim x_{0}}=\frac{1}{2} \tilde{\nabla} \log \left(w^{\langle A\rangle}\right)^{2}=\boldsymbol{m}_{A} .
$$

This implies that the region where the weight $w^{\langle A\rangle}$ is dominant corresponds to the vacuum $\langle A\rangle$.

Let us next consider a situation that the vacua $\langle A\rangle$ and $\langle B\rangle$ are next to each other. We can estimate where the two vacua transit by comparing the weights of those vacua. The transition occurs at points on which the two weights are equal. This condition determines a hyperplane which is a subspace of codimension 1 in $\mathbb{R}^{D}$,

$$
\langle A, B\rangle: w^{\langle A\rangle}=w^{\langle B\rangle} \Leftrightarrow\left(\tilde{\boldsymbol{m}}_{A}-\tilde{\boldsymbol{m}}_{B}\right) \cdot \boldsymbol{x}+a_{A}-a_{B}=0 .
$$

This is a straightforward generalization of $D=2$ [75] to generic $D$ dimensions. This hyperplane is nothing but a domain wall interpolating the vacua $\langle A\rangle$ and $\langle B\rangle$, and we call it a 1-wall.

The three vacua, say $\langle A\rangle,\langle B\rangle$, and $\langle C\rangle$, can happen to be adjacent at a hyperplane of codimension 2 , which is conventionally called the domain wall junction. We call it a 2-wall. The position of the 2-wall corresponds to the region where the weights are equal as

$$
\langle A, B, C\rangle: w^{\langle A\rangle}=w^{\langle B\rangle}=w^{\langle C\rangle} .
$$

These can be naturally generalized to a $d$ wall which is a $d$ codimensional intersection dividing $d+1$ vacua. The position of the $d$ wall is defined by

$$
\left\langle A_{1}, A_{2}, \ldots, A_{d+1}\right\rangle: w^{\left\langle A_{1}\right\rangle}=w^{\left\langle A_{2}\right\rangle}=\cdots=w^{\left\langle A_{d+1}\right\rangle} .
$$

In the next section, we will see that the position of the $d$ wall estimated by the weight is related to the generalized topological charge $\mathcal{W}_{d}$ defined in Eq. (3.14).

\section{EXHAUSTING ALL EXACT SOLUTIONS OF DOMAIN WALL NETWORKS IN THE $\mathbb{C} P^{N-1}$ MODEL}

\section{A. General solutions}

There is a great simplification allowing us to obtain all exact solutions for generic domain wall networks in $D$ dimensions. It is the infinite gauge coupling limit in which we formally send the gauge coupling $e$ to infinity in the Lagrangian (2.1). There, the kinetic terms of $A_{\mu}$ and $\Sigma_{m}$ vanish to become Lagrange multipliers. At the same time, the first term in the potential (2.2) forces the charged fields $H^{A}$ to take their values in the restricted region $S^{2 N-1}$ defined by $H H^{\dagger}=v^{2}$. Furthermore, the overall phase of $H_{A}$ is gauged. Therefore, the physical target space in the infinite gauge coupling limit is reduced to the complex projective space,

$$
\mathbb{C} P^{N-1} \simeq \frac{S U(N)}{S U(N-1) \times U(1)} \simeq \frac{S^{2 N-1}}{S^{1}} .
$$

Indeed, if we eliminate the gauge field $A_{\mu}$ from $\left.\mathcal{L}\right|_{e \rightarrow \infty}$, it reduces to the standard Lagrangian of the nonlinear $\mathbb{C} P^{N-1}$ model. Similarly, if we eliminate the neutral scalar fields $\Sigma_{m}$, we get a nontrivial potential which lifts all the points of the $\mathbb{C} P^{N-1}$ target space leaving $N$ discrete points as vacua.

Here, we do not eliminate the auxiliary fields $A_{\mu}$ and $\Sigma_{m}$. The BPS equations in Eqs. (3.10)-(3.13) remain the same, and the first three equations (3.10)-(3.12) are solved by Eqs. (3.15) and (3.17). The fourth equation (3.13) rewritten as Eq. (3.19) reduces to an algebraic equation, easily solved by

$$
\left.\Omega\right|_{e \rightarrow \infty}=H_{0} e^{2 \xi_{m} M_{m} x^{m}} H_{0}^{\dagger} .
$$

Thus, we have completely solved the BPS equations for arbitrary moduli matrix $H_{0}$ in the infinite gauge coupling limit. We would like to emphasize that this is the first solutions of the domain wall networks in $D \geq 3$.

For later convenience, let us rewrite this in a more useful form. First, let us denote $H_{0}$ as

$$
H_{0}=\left(e^{a_{1}+i b_{1}}, e^{a_{2}+i b_{2}}, \ldots, e^{a_{N}+i b_{N}}\right),
$$

where $\left\{a_{A}\right\}$ and $\left\{b_{A}\right\}$ are $N$ real parameters which we restrict to satisfy the conditions $\sum_{A} a_{A}=\sum_{A} b_{A}=0$ by using the $V$ transformation. Furthermore, let us define the new mass vectors

$$
\tilde{\boldsymbol{m}}_{A}=\left(\xi_{1} m_{1, A}, \boldsymbol{\xi}_{2} m_{2, A}, \ldots, \boldsymbol{\xi}_{D} m_{D, A}\right) .
$$


Then, we have

$$
\left.\Omega\right|_{e \rightarrow \infty}=\sum_{A=1}^{N} e^{2\left(\tilde{\boldsymbol{m}}_{A} \cdot \boldsymbol{x}+a_{A}\right)} .
$$

By using this, the BPS energy density can be simply expressed by

$\left.\mathcal{E}\right|_{e \rightarrow \infty}=v^{2} \tilde{\nabla} \cdot \boldsymbol{\Sigma}=\frac{v^{2}}{2} \nabla^{2} \log \left(\sum_{A=1}^{N} e^{2\left(\tilde{\boldsymbol{m}}_{A} \cdot \boldsymbol{x}+a_{A}\right)}\right)$,

where we have defined

$$
\tilde{\nabla}=\left(\xi_{1} \partial_{1}, \xi_{2} \partial_{2}, \ldots, \xi_{D} \partial_{D}\right), \quad \tilde{\nabla}^{2}=\nabla^{2}
$$

and used $\boldsymbol{\Sigma}=\frac{1}{2} \tilde{\nabla} \log \Omega$. From this expression, we can see that the $N-1$ real parameters $\left\{a_{A}\right\}$ are the moduli which relate to the shape of the networks in the real space. On the other hand, the other parameters $\left\{b_{A}\right\}$ are internal moduli of $U(1)^{N-1}$ associated with constituent domain walls.

The domain walls for $D=1$ and the domain wall networks for $D=2$ have been studied very well in the literature; therefore, we will concentrate on $D=3$ in the following subsections.

\section{B. $N=4$ : Tetrahedron: Single domain wall junction}

Let us start with a model with $N=4$. There are $N=4$ vacua which are the minimal numbers for a nonplanar domain wall junction to exist. For simplicity, let us set the four mass vectors $\boldsymbol{m}_{A}$ to be four vertices of a regular tetrahedron as

$$
\begin{gathered}
\boldsymbol{m}_{1}=\left(-\frac{1}{\sqrt{3}},-1,-\frac{1}{\sqrt{6}}\right), \\
\boldsymbol{m}_{2}=\left(\frac{2}{\sqrt{3}}, 0,-\frac{1}{\sqrt{6}}\right), \\
\boldsymbol{m}_{3}=\left(-\frac{1}{\sqrt{3}}, 1,-\frac{1}{\sqrt{6}}\right), \\
\boldsymbol{m}_{4}=\left(0,0, \sqrt{\frac{3}{2}}\right) .
\end{gathered}
$$

Then, the four vacua correspond to four vertices of the regular tetrahedron in the $\Sigma_{1}-\Sigma_{2}-\Sigma_{3}$ space, as shown in Fig. 1. We call polyhedrons drawn in the $\boldsymbol{\Sigma}$ space as grid diagrams [75]. Note that we have taken the regular tetrahedron (4.8)-(4.11) just for simplicity. In general, the grid diagrams do not have to be congruent with a regular tetrahedron. The following arguments are valid for the generic grid diagrams.

As we explained in Eq. (3.25), single domain walls (1-walls) can be described by the moduli matrix with nonvanishing elements. For instance, the moduli matrix $H_{0}=\left(e^{a+i b}, e^{-a-i b}, 0,0\right)$ yields a domain wall $\langle 1,2\rangle$ connecting the $\langle 1\rangle$ and $\langle 2\rangle$ vacua. The corresponding $\Omega$ is

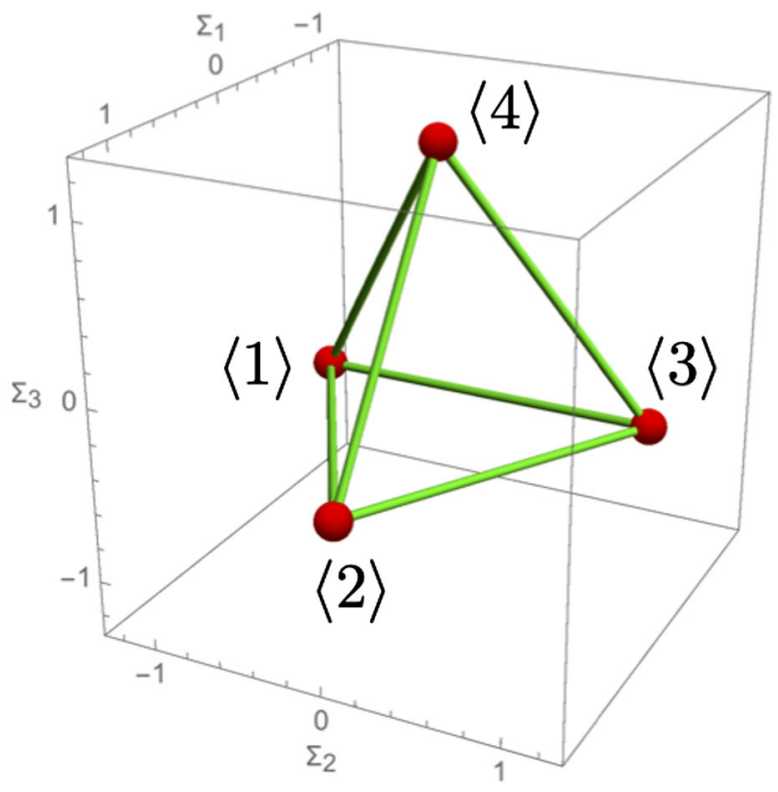

FIG. 1. The grid diagram ( $\boldsymbol{\Sigma}$ space): the four vacua (red points) in $N=4$ model are shown.

$\Omega=w^{\langle 1\rangle}+w^{\langle 2\rangle}$, and the exact domain wall solution is given as

$$
\begin{aligned}
& \Sigma_{1}=\frac{m_{1,1} e^{2\left(\tilde{\boldsymbol{m}}_{1} \cdot \boldsymbol{x}+a\right)}+m_{1,2} e^{2\left(\tilde{\boldsymbol{m}}_{2} \cdot \boldsymbol{x}-a\right)}}{e^{2\left(\tilde{\boldsymbol{m}}_{1} \cdot \boldsymbol{x}-a\right)}+e^{2\left(\tilde{\boldsymbol{m}}_{2} \cdot \boldsymbol{x}-a\right)}}, \\
& \Sigma_{2}=\frac{m_{2,1} e^{2\left(\tilde{\boldsymbol{m}}_{1} \cdot \boldsymbol{x}+a\right)}+m_{2,2} e^{2\left(\tilde{\boldsymbol{m}}_{2} \cdot \boldsymbol{x}-a\right)}}{e^{2\left(\tilde{\boldsymbol{m}}_{1} \cdot \boldsymbol{x}-a\right)}+e^{2\left(\tilde{\boldsymbol{m}}_{2} \cdot \boldsymbol{x}-a\right)}}, \\
& \Sigma_{3}=\frac{m_{3,1} e^{2\left(\tilde{\boldsymbol{m}}_{1} \cdot \boldsymbol{x}+a\right)}+m_{3,2} e^{2\left(\tilde{\boldsymbol{m}}_{2} \cdot \boldsymbol{x}-a\right)}}{e^{2\left(\tilde{\boldsymbol{m}}_{1} \cdot \boldsymbol{x}-a\right)}+e^{2\left(\tilde{\boldsymbol{m}}_{2} \cdot \boldsymbol{x}-a\right)}} .
\end{aligned}
$$

Note that $\Sigma_{i}$ takes its value in the finite interval $\Sigma_{i} \in$ $\left[m_{i, 1}, m_{i, 2}\right](i=1,2)$ when we sweep the real space $\mathbb{R}^{3}$. Namely, $\boldsymbol{\Sigma}$ connects the two vertices $\boldsymbol{m}_{1}$ and $\boldsymbol{m}_{2}$, as desired. Thus, the domain wall solution $\boldsymbol{\Sigma}(\boldsymbol{x})$ can be seen as a function from the real space $\mathbb{R}^{3}$ to the $\boldsymbol{\Sigma}$ space, and its image is the linear segment between $\boldsymbol{m}_{1}$ and $\boldsymbol{m}_{\mathbf{2}}$,

$$
\frac{\Sigma_{1}-m_{1,1}}{\Sigma_{1}-m_{1,2}}=\frac{\Sigma_{2}-m_{2,1}}{\Sigma_{2}-m_{2,2}}=\frac{\Sigma_{3}-m_{3,1}}{\Sigma_{3}-m_{3,2}} .
$$

The topological charges of 1-walls associated with Eq. (3.14) are

$W_{1}^{(x)}\left(\boldsymbol{m}_{1}, \boldsymbol{m}_{2}\right)=\int_{-\infty}^{\infty} d x \partial_{1} \Sigma_{1}=\int d \Sigma_{1}=\left|m_{1,1}-m_{1,2}\right|$,

$W_{1}^{(y)}\left(\boldsymbol{m}_{1}, \boldsymbol{m}_{2}\right)=\int_{-\infty}^{\infty} d y \partial_{2} \Sigma_{2}=\int d \Sigma_{2}=\left|m_{2,1}-m_{2,2}\right|$, 


$$
W_{1}^{(z)}\left(\boldsymbol{m}_{1}, \boldsymbol{m}_{2}\right)=\int_{-\infty}^{\infty} d z \partial_{3} \Sigma_{3}=\int d \Sigma_{3}=\left|m_{3,1}-m_{3,2}\right| .
$$

These are the lengths of segments of the edge connecting $\langle 1\rangle$ and $\langle 2\rangle$ projected onto the axes $\Sigma_{1,2,3}$. It is straightforward to construct the other domain walls connecting arbitrary pair of $\langle A\rangle$ and $\langle B\rangle$. They correspond to the edges of the tetrahedron in Fig. 1.

A domain wall junction (2-wall) connecting three vacua, say $\langle 1\rangle,\langle 2\rangle$, and $\langle 3\rangle$, can be also constructed very easily. One mere needs to prepare the moduli matrix $H_{0}=$ $\left(e^{a_{1}+i b_{1}}, e^{a_{2}+i b_{2}}, e^{a_{3}+i b_{3}}, 0\right)$ with three nonzero elements. The exact solution is given by

$$
\Sigma_{1}=\frac{m_{1,1} e^{2\left(\tilde{\boldsymbol{m}}_{1} \cdot \boldsymbol{x}+a_{1}\right)}+m_{1,2} e^{2\left(\tilde{\boldsymbol{m}}_{2} \cdot \boldsymbol{x}+a_{2}\right)}+m_{1,3} e^{2\left(\tilde{\boldsymbol{m}}_{3} \cdot \boldsymbol{x}+a_{3}\right)}}{e^{2\left(\tilde{\boldsymbol{m}}_{1} \cdot \boldsymbol{x}+a_{1}\right)}+e^{2\left(\tilde{\boldsymbol{m}}_{2} \cdot \boldsymbol{x}+a_{2}\right)}+e^{2\left(\tilde{\boldsymbol{m}}_{3} \cdot \boldsymbol{x}+a_{3}\right)}},
$$

$$
\Sigma_{2}=\frac{m_{2,1} e^{2\left(\tilde{\boldsymbol{m}}_{1} \cdot \boldsymbol{x}+a_{1}\right)}+m_{2,2} e^{2\left(\tilde{\boldsymbol{m}}_{2} \cdot \boldsymbol{x}+a_{2}\right)}+m_{2,3} e^{2\left(\tilde{\boldsymbol{m}}_{3} \cdot \boldsymbol{x}+a_{3}\right)}}{e^{2\left(\tilde{\boldsymbol{m}}_{1} \cdot \boldsymbol{x}+a_{1}\right)}+e^{2\left(\tilde{\boldsymbol{m}}_{2} \cdot \boldsymbol{x}+a_{2}\right)}+e^{2\left(\tilde{\boldsymbol{m}}_{3} \cdot \boldsymbol{x}+a_{3}\right)}},
$$

$$
\Sigma_{3}=\frac{m_{3,1} e^{2\left(\tilde{\boldsymbol{m}}_{1} \cdot \boldsymbol{x}+a_{1}\right)}+m_{3,2} e^{2\left(\tilde{\boldsymbol{m}}_{2} \cdot \boldsymbol{x}+a_{2}\right)}+m_{3,3} e^{2\left(\tilde{\boldsymbol{m}}_{3} \cdot \boldsymbol{x}+a_{3}\right)}}{e^{2\left(\tilde{\boldsymbol{m}}_{1} \cdot \boldsymbol{x}+a_{1}\right)}+e^{2\left(\tilde{\boldsymbol{m}}_{2} \cdot \boldsymbol{x}+a_{2}\right)}+e^{2\left(\tilde{\boldsymbol{m}}_{3} \cdot \boldsymbol{x}+a_{3}\right)}} .
$$

These $\boldsymbol{\Sigma}$ satisfy the equation

$$
\left\{\left(\boldsymbol{m}_{3}-\boldsymbol{m}_{1}\right) \times\left(\boldsymbol{m}_{2}-\boldsymbol{m}_{1}\right)\right\} \cdot\left(\boldsymbol{\Sigma}-\boldsymbol{m}_{1}\right)=\mathbf{0},
$$

representing the two-dimensional plane on which the three points $\boldsymbol{m}_{1}, \boldsymbol{m}_{2}$, and $\boldsymbol{m}_{3}$ are located. The image of the map $\boldsymbol{\Sigma}(\boldsymbol{x})$ in this case is the triangle whose vertices are $\langle 1\rangle,\langle 2\rangle$, and $\langle 3\rangle$. The corresponding topological charges are identical to the areas of the projections of the triangle onto the three planes $\left(\Sigma_{1}-\Sigma_{2}, \Sigma_{2}-\Sigma_{3}\right.$, and $\left.\Sigma_{3}-\Sigma_{1}\right)$ as

$$
\begin{aligned}
W_{2}^{(x y)}\left(\boldsymbol{m}_{1}, \boldsymbol{m}_{2}, \boldsymbol{m}_{3}\right) & =\int_{-\infty}^{\infty} d x d y\left(\partial_{1} \Sigma_{2} \partial_{2} \Sigma_{1}-\partial_{1} \Sigma_{1} \partial_{2} \Sigma_{2}\right) \\
& =\int d \Sigma_{1} d \Sigma_{2}=\frac{1}{2}\left|\left[\left(\boldsymbol{m}_{1}-\boldsymbol{m}_{2}\right) \times\left(\boldsymbol{m}_{1}-\boldsymbol{m}_{3}\right)\right]_{3}\right|, \\
W_{2}^{(y z)}\left(\boldsymbol{m}_{1}, \boldsymbol{m}_{2}, \boldsymbol{m}_{3}\right) & =\int_{-\infty}^{\infty} d y d z\left(\partial_{2} \Sigma_{3} \partial_{3} \Sigma_{2}-\partial_{2} \Sigma_{2} \partial_{3} \Sigma_{3}\right) \\
& =\int d \Sigma_{2} d \Sigma_{3}=\frac{1}{2}\left|\left[\left(\boldsymbol{m}_{1}-\boldsymbol{m}_{2}\right) \times\left(\boldsymbol{m}_{1}-\boldsymbol{m}_{3}\right)\right]_{1}\right|, \\
W_{2}^{(z x)}\left(\boldsymbol{m}_{1}, \boldsymbol{m}_{2}, \boldsymbol{m}_{3}\right) & =\int_{-\infty}^{\infty} d z d x\left(\partial_{3} \Sigma_{1} \partial_{1} \Sigma_{3}-\partial_{3} \Sigma_{3} \partial_{1} \Sigma_{1}\right) \\
& =\int d \Sigma_{3} d \Sigma_{1}=\frac{1}{2}\left|\left[\left(\boldsymbol{m}_{1}-\boldsymbol{m}_{2}\right) \times\left(\boldsymbol{m}_{1}-\boldsymbol{m}_{3}\right)\right]_{2}\right| .
\end{aligned}
$$

Again, it is straightforward to construct the other 2-walls dividing arbitrary set of three vacua. They correspond to the faces of the tetrahedron in Fig. 1.

Finally, we come to the 3 -wall $\langle 1,2,3,4\rangle$ consisting of the four vacua, six 1-walls, and four 2-walls. It is described by the full moduli matrix $H_{0}=\left(e^{a_{1}+i b_{1}}, e^{a_{2}+i b_{2}}, e^{a_{3}+i b_{3}}, e^{a_{4}+i b_{4}}\right)$, and the exact solution is given by

$$
\begin{aligned}
& \Sigma_{1}=\frac{m_{1,1} e^{2\left(\tilde{\boldsymbol{m}}_{1} \cdot \boldsymbol{x}+a_{1}\right)}+m_{1,2} e^{2\left(\tilde{\boldsymbol{m}}_{2} \cdot \boldsymbol{x}+a_{2}\right)}+m_{1,3} e^{2\left(\tilde{\boldsymbol{m}}_{3} \cdot \boldsymbol{x}+a_{3}\right)}+m_{1,4} e^{2\left(\tilde{\boldsymbol{m}}_{4} \cdot \boldsymbol{x}+a_{4}\right)}}{e^{2\left(\tilde{\boldsymbol{m}}_{1} \cdot \boldsymbol{x}+a_{1}\right)}+e^{2\left(\tilde{\boldsymbol{m}}_{2} \cdot \boldsymbol{x}+a_{2}\right)}+e^{2\left(\tilde{\boldsymbol{m}}_{3} \cdot \boldsymbol{x}+a_{3}\right)}+e^{2\left(\tilde{\boldsymbol{m}}_{4} \cdot \boldsymbol{x}+a_{4}\right)}}, \\
& \Sigma_{2}=\frac{m_{2,1} e^{2\left(\tilde{\boldsymbol{m}}_{1} \cdot \boldsymbol{x}+a_{1}\right)}+m_{2,2} e^{2\left(\tilde{\boldsymbol{m}}_{2} \cdot \boldsymbol{x}+a_{2}\right)}+m_{2,3} e^{2\left(\tilde{\boldsymbol{m}}_{3} \cdot \boldsymbol{x}+a_{3}\right)}+m_{2,4} e^{2\left(\tilde{\boldsymbol{m}}_{4} \cdot \boldsymbol{x}+a_{4}\right)}}{e^{2\left(\tilde{\boldsymbol{m}}_{1} \cdot \boldsymbol{x}+a_{1}\right)}+e^{2\left(\tilde{\boldsymbol{m}}_{2} \cdot \boldsymbol{x}+a_{2}\right)}+e^{2\left(\tilde{\boldsymbol{m}}_{3} \cdot \boldsymbol{x}+a_{3}\right)}+e^{2\left(\tilde{\boldsymbol{m}}_{4} \cdot \boldsymbol{x}+a_{4}\right)}}, \\
& \Sigma_{3}=\frac{m_{3,1} e^{2\left(\tilde{\boldsymbol{m}}_{1} \cdot \boldsymbol{x}+a_{1}\right)}+m_{3,2} e^{2\left(\tilde{\boldsymbol{m}}_{2} \cdot \boldsymbol{x}+a_{2}\right)}+m_{3,3} e^{2\left(\tilde{\boldsymbol{m}}_{3} \cdot \boldsymbol{x}+a_{3}\right)}+m_{3,4} e^{2\left(\tilde{\boldsymbol{m}}_{4} \cdot \boldsymbol{x}+a_{4}\right)}}{e^{2\left(\tilde{\boldsymbol{m}}_{1} \cdot \boldsymbol{x}+a_{1}\right)}+e^{2\left(\tilde{\boldsymbol{m}}_{2} \cdot \boldsymbol{x}+a_{2}\right)}+e^{2\left(\tilde{\boldsymbol{m}}_{3} \cdot \boldsymbol{x}+a_{3}\right)}+e^{2\left(\tilde{\boldsymbol{m}}_{4} \cdot \boldsymbol{x}+a_{4}\right)}} .
\end{aligned}
$$

Note that we can set $a_{1}=a_{2}=a_{3}=a_{4}$ by using three translational symmetries and $V$ transformation, without loss of generality. Now, the solution $\boldsymbol{\Sigma}(\boldsymbol{x})$ maps the real three-dimensional space $\mathbb{R}^{3}$ to the tetrahedron itself in the $\boldsymbol{\Sigma}$ space. The corresponding topological charge reads 


$$
\begin{aligned}
W_{3}^{(x y z)}\left(\boldsymbol{m}_{1}, \boldsymbol{m}_{2}, \boldsymbol{m}_{3}, \boldsymbol{m}_{4}\right) & =\int_{-\infty}^{\infty} d x d y d z \operatorname{det}\left(\begin{array}{ccc}
\partial_{1} \Sigma_{1} & \partial_{1} \Sigma_{2} & \partial_{1} \Sigma_{3} \\
\partial_{2} \Sigma_{1} & \partial_{2} \Sigma_{2} & \partial_{2} \Sigma_{3} \\
\partial_{3} \Sigma_{1} & \partial_{3} \Sigma_{2} & \partial_{3} \Sigma_{3}
\end{array}\right)=\int d \Sigma_{1} d \Sigma_{2} d \Sigma_{3} \\
& \left.=\frac{1}{6} \mid\left(\left(\boldsymbol{m}_{2}-\boldsymbol{m}_{1}\right) \times\left(\boldsymbol{m}_{3}-\boldsymbol{m}_{1}\right)\right) \cdot\left(\boldsymbol{m}_{4}-\boldsymbol{m}_{1}\right)\right) \mid .
\end{aligned}
$$

This is nothing but the volume of the tetrahedron.

In Fig. 2, we show the topological charge densities $\mathcal{W}_{d}$ for the regular tetrahedron given in Fig. 1. The top-left panel shows the vacuum ( 0 -wall) domains $(\langle 1\rangle=$ green, $\langle 2\rangle=$ yellow, $\langle 3\rangle=$ cyan, $\langle 1\rangle=$ hidden $)$. At boundaries between two vacuum ( 0 -wall) domains, there are domain walls (1-walls), as shown in the top-right panel. The plot shows an isosurface of the sum of three 1-wall charge densities $\mathcal{W}_{1}^{(x)}+\mathcal{W}_{1}^{(y)}+\mathcal{W}_{1}^{(z)}$. Similarly, the bottom-left panel shows an isosurface of the sum of the three 2-wall charge densities $\mathcal{W}_{2}^{(x y)}+\mathcal{W}_{2}^{(y z)}+\mathcal{W}_{2}^{(z x)}$. Finally, the bottom-right panel shows an isosurface of the 3-wall charge density $\mathcal{W}_{3}^{(x y z)}$. The figures clearly show that the generalized topological charge defined in Eq. (3.14) is appropriate to describe the codimension $d$ structure in the solution.

To close this subsection, we again emphasize that we have chosen the symmetric arrangement of the masses corresponding to a regular tetrahedron in Fig. 1 just for simplicity, but the exact solution has been obtained for arbitrary mass arrangement. In order to make this point clearer, we show in Fig. 3 four solutions for randomly chosen tetrahedrons.
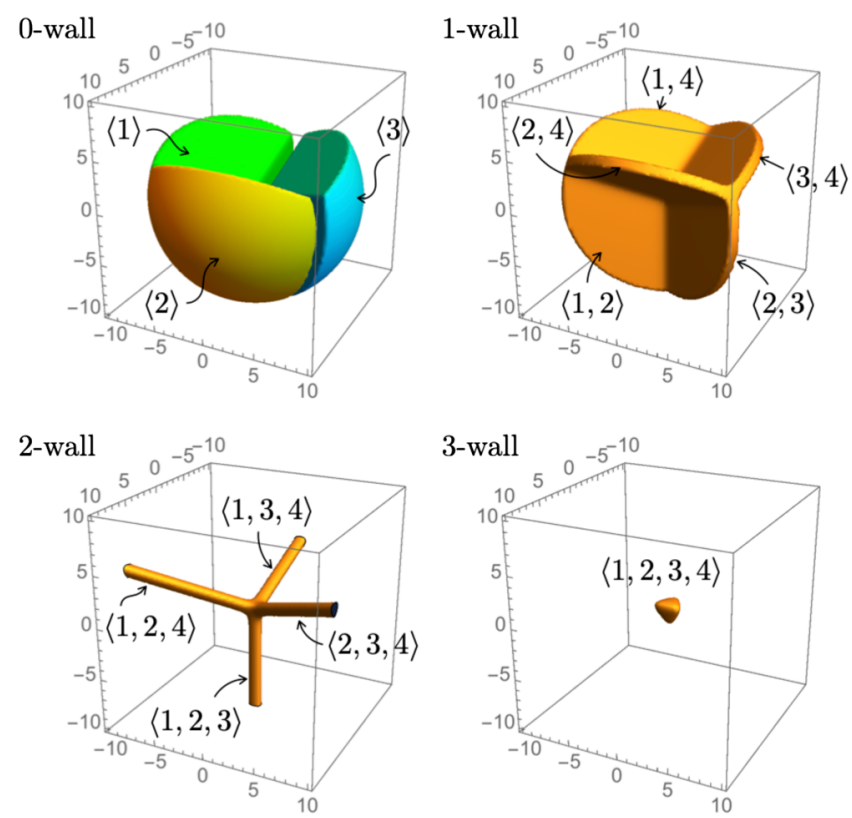

FIG. 2. The real space $\mathbb{R}^{3}$ : isosurfaces of the topological charge densities $\mathcal{W}_{d}$ for $d$ walls $(d=0,1,2,3)$ are shown. We only depict the densities within the sphere of the radius 10 .

\section{C. $N=5$ : Dipyramid: Minimal domain wall networks}

Let us next consider a model admitting a nonplanar network structure of domain walls. The minimal number for this is $N=5$ for which there is one additional vacuum $\langle 5\rangle$ compared to the model with $N=4$. According to where we put the fifth vacuum, the resulting network structures are classified into two types as shown in Figs. 4(a) and 4(b). The feature of (a) is the presence of an inner vacuum $\langle 5\rangle$ in the tetrahedron $\langle 1,2,3,4\rangle$. On the other hand, Fig. 4(b) is a dipyramid for which the fifth vacuum $\langle 5\rangle$ is placed outside the tetrahedron $\langle 1,2,3,4\rangle$ It is a convex polytope and has no inner vacua.

Let us start with the former case of the tetrahedron with the inner vacuum. We take the same four vacua defined in Eqs. (4.8)-(4.11) for simplicity, and the fifth one is set as

$$
\boldsymbol{m}_{5}=(0,0,0) \text {. }
$$

The most generic moduli matrix up to the three translations in $\mathbb{R}^{3}$ and $V$ transformation (3.20) is given by

$$
H_{0}=\left(1,1,1,1, e^{a_{5}}\right) .
$$

Then $\Omega$ depends on the one moduli parameter $a_{5}$ as

$$
\Omega=\sum_{A=1}^{4} e^{2 \tilde{\boldsymbol{m}}_{A} \cdot \boldsymbol{x}}+e^{2 a_{5}} e^{2 \tilde{\boldsymbol{m}}_{5} \cdot \boldsymbol{x}} .
$$

From this expression, we understand that the factor $e^{a_{5}}$ controls the strength (the weight) of the fifth vacuum $\langle 5\rangle$ relative to the rests. When $a_{5} \rightarrow-\infty$, the $\langle 5\rangle$ domain disappears. The $\langle 5\rangle$ domain extends as $e^{a_{5}}$ increases. This moduli dependence of the configuration can be seen in Fig. 5 in which we have shown three examples with $a_{5}=-\infty, 0$, and 3 . The large distance behaviors are the same for all these cases. However, a significant difference emerges around the origin. Reflecting the inner vacuum $\langle 5\rangle$ inside the tetrahedron, a compact vacuum domain of $\langle 5\rangle$ emerges by increasing $a_{5}$. The whole structure of the solution is as follows. There is one inner vacuum bubble $\langle 5\rangle$ surrounded by the semi-infinite four vacuum domains $\langle 1\rangle,\langle 2\rangle,\langle 3\rangle$, and $\langle 4\rangle$. There are six semi-infinite 1-walls corresponding to six outer edges $(\langle 1,2\rangle,\langle 1,3\rangle,\langle 1,4\rangle,\langle 2,3\rangle$, $\langle 2,4\rangle$, and $\langle 3,4\rangle)$ and four compact 1 -walls corresponding to the four inner edges $(\langle 1,5\rangle,\langle 2,5\rangle,\langle 3,5\rangle,\langle 4,5\rangle)$; see the third row of Fig. 5. There are four semi-infinite 2 -walls 

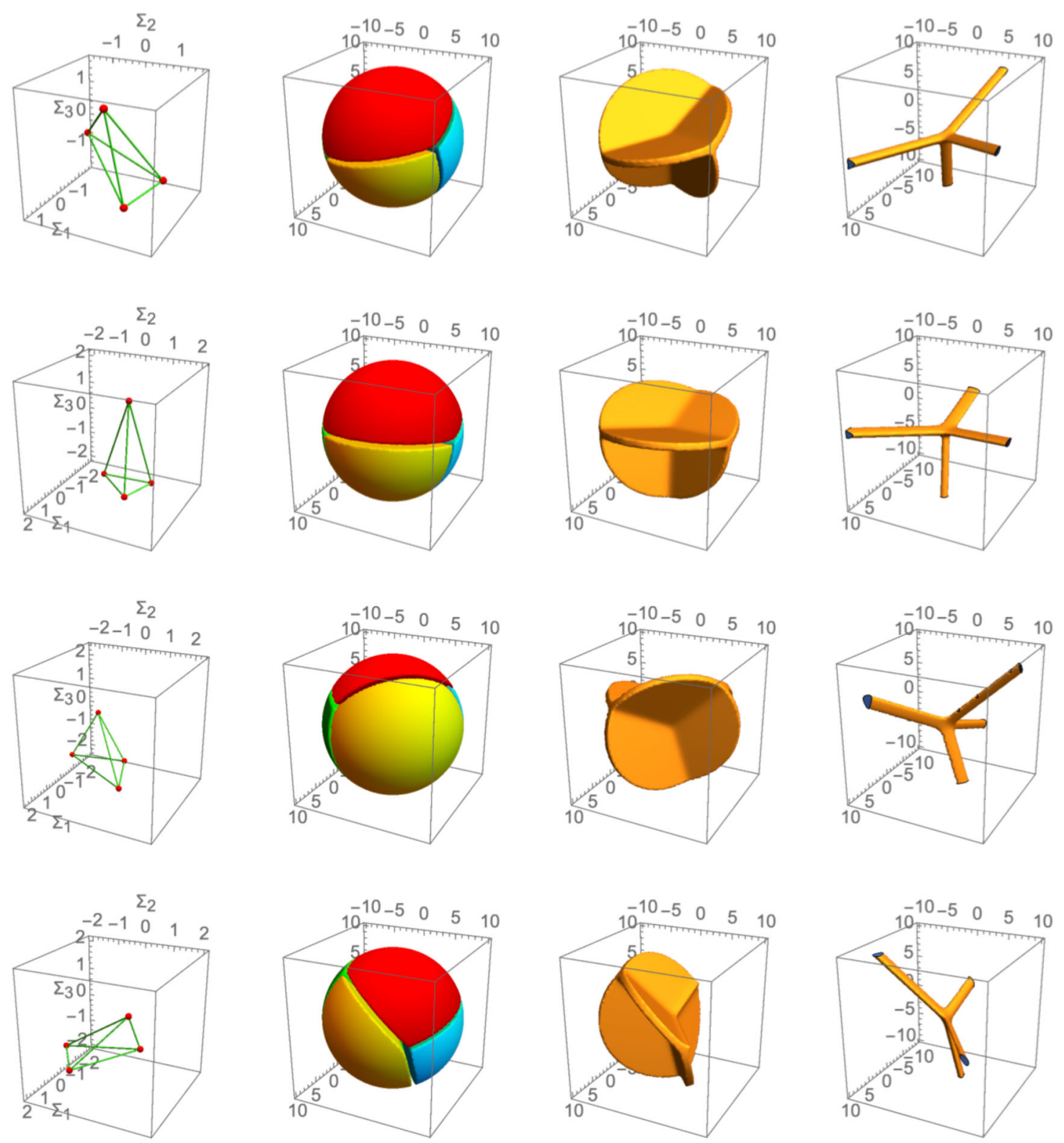

FIG. 3. Four examples of the BPS solutions: the leftmost panel shows the grid diagrams, and the second, third, and fourth from left show the corresponding $\mathcal{W}_{d} d=0,1,2$. We only depict the densities within the sphere of the radius 10 .

and the six compact 2-walls corresponding to the four faces $(\langle 1,2,3\rangle,\langle 1,2,4\rangle,\langle 1,3,4\rangle,\langle 2,3,4\rangle)$ and the six inner triangles $(\langle 1,2,5\rangle,\langle 2,3,5\rangle,\langle 1,3,5\rangle,\langle 1,4,5\rangle,\langle 2,4,5\rangle$, $\langle 3,4,5\rangle)$, respectively. Finally, there are four 3 -walls which correspond to the four subtetrahedrons $(\langle 1,2,4,5\rangle$, $\langle 2,3,4,5\rangle,\langle 1,3,4,5\rangle,\langle 1,2,3,5\rangle)$. The network structure in the real $\mathbb{R}^{3}$ space can be best seen in the plot of the 2-walls as shown in Fig. 5.

Next, let us place the fifth vacuum $\langle 5\rangle$ on the plane including the bottom triangle $\langle 1,2,3\rangle$, say

$$
\boldsymbol{m}_{5}=\left(0,0,-\frac{1}{\sqrt{6}}\right)
$$

The moduli matrix is the same as the one in Eq. (4.31). When $e^{a_{5}}$ is sufficiently large, the configuration becomes a mixture of planar and nonplanar structures, as shown in Fig. 6 for $a_{5}=3$. The planar structure comes from the bottom triangle $\langle 1,2,3\rangle$ which are divided into three subtriangles $\langle 1,2,5\rangle,\langle 2,3,5\rangle$, and $\langle 1,3,5\rangle$. As a consequence, there are six parallel semi-infinite 1-walls corresponding to $\langle 1,2\rangle,\langle 1,3\rangle,\langle 1,5\rangle,\langle 2,3\rangle,\langle 2,5\rangle$, and $\langle 3,5\rangle$. On the other hand, the nonplanar structure is originated from the vacuum $\langle 4\rangle$ which is placed off the bottom triangle. In addition to the semi-infinite 1 -walls corresponding to the edges $\langle 1,4\rangle,\langle 2,4\rangle,\langle 3,4\rangle$, there is the compact 1 -wall of $\langle 4,5\rangle$. Comparing the 2 - and 3 -wall structures in Figs. 5 and 6, we see that the lower 3-walls is pushed down 


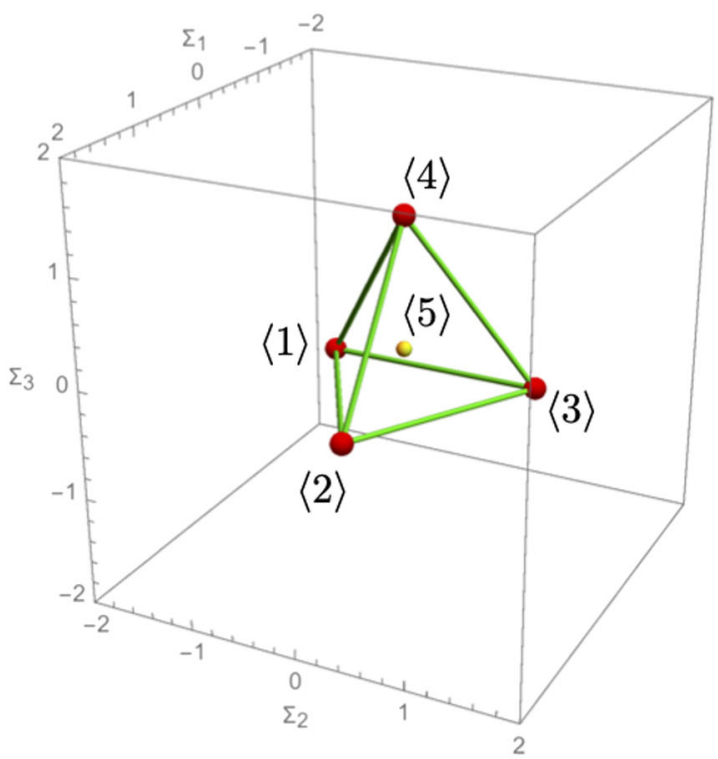

(a)

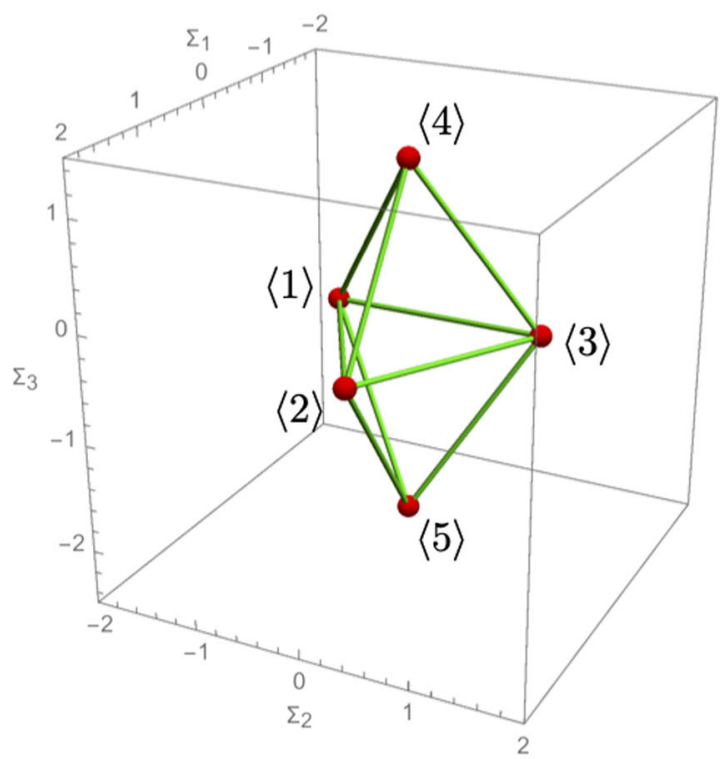

(b)

FIG. 4. The grid diagrams ( $\boldsymbol{\Sigma}$ space): (a),(b) show the two different patterns of five vacua in $N=5$.

toward infinity, which corresponds to the fact that the subtetrahedron $\langle 1,2,3,5\rangle$ gets squashed flat.

Let us next study the second type with the dypyramid structure as Fig. 4(b), which can be obtained by further pushing down the vacuum $\langle 5\rangle$ from the last case in Fig. 6. As before, the first four masses $\boldsymbol{m}_{1}, \boldsymbol{m}_{2}, \boldsymbol{m}_{3}$, and $\boldsymbol{m}_{4}$ are intact. Then the fifth vacuum is placed at opposite to $\langle 4\rangle$ with respect to the triangle $\langle 1,2,3\rangle$ as

$$
\boldsymbol{m}_{5}=\left(0,0,-\frac{5}{\sqrt{6}}\right)
$$

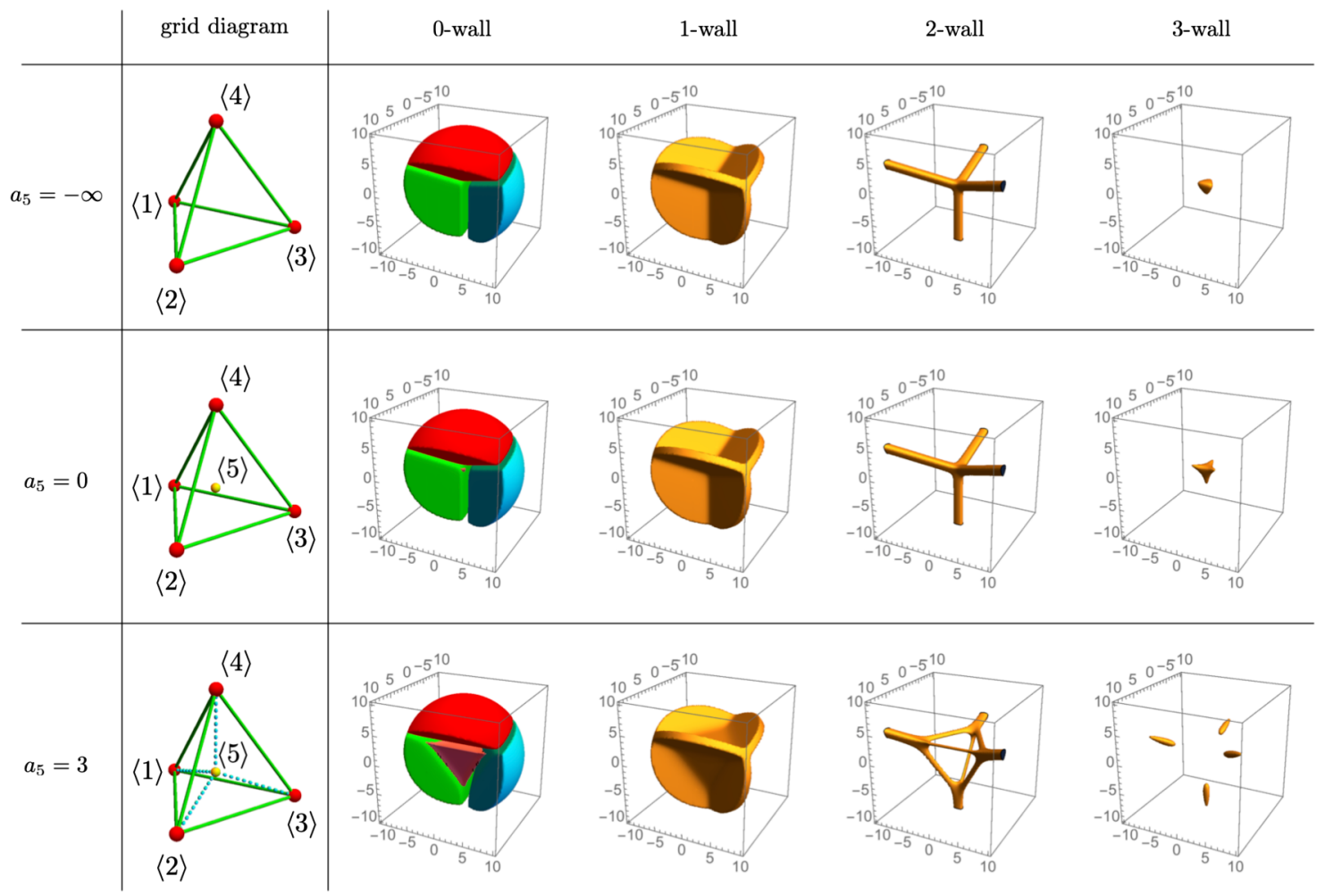

FIG. 5. Exact solutions in three typical branches for the grid diagram in Fig. 4(a). Isosurfaces of the topological charge densities $\mathcal{W}_{d}$ for $d$ walls $(d=0,1,2,3)$ are shown. 


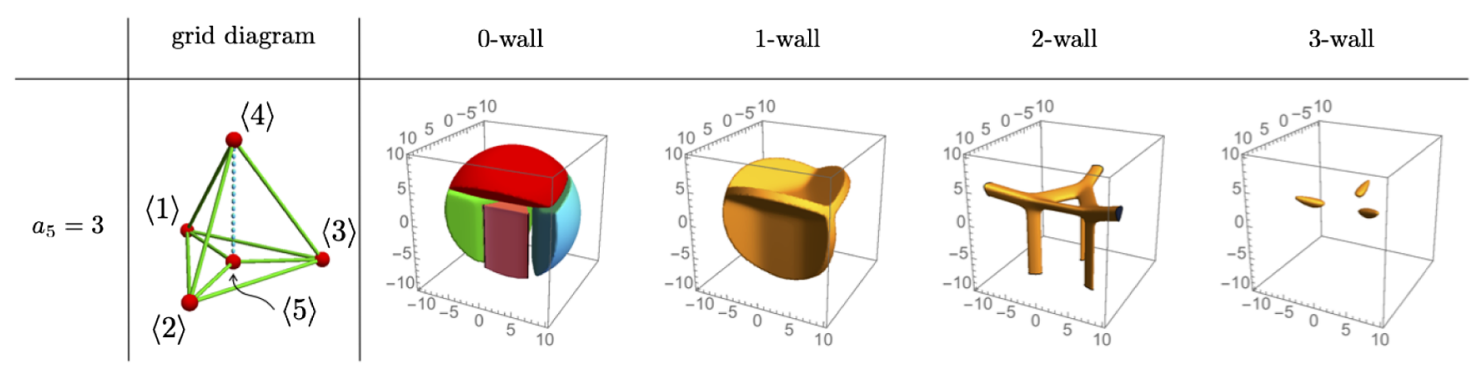

FIG. 6. Exact solution for the fine-tuned grid diagram. Isosurfaces of the topological charge densities $\mathcal{W}_{d}$ for $d$ walls $(d=0,1,2,3)$ are shown.

A useful choice of the moduli matrix with one moduli parameter $a_{45}$ is given by

$$
H_{0}=\left(1,1,1, e^{a_{45}}, e^{a_{45}}\right) .
$$

The parameter $a_{45}$ controls the weight of the vacua $\langle 4\rangle$ and $\langle 5\rangle$ relative to that of $\langle 1\rangle,\langle 2\rangle$, and $\langle 3\rangle$. We show three typical solutions with $a_{45}=-4,0,3$ in Fig. 7 .

The first row corresponds to $a_{45}=-4$. The $\langle 4\rangle$ and $\langle 5\rangle$ domains are relatively weak, whereas the domains of $\langle 1\rangle$, $\langle 2\rangle$, and $\langle 3\rangle$ stick out and directly meet to form a 2 -wall corresponding to the inner triangle $\langle 1,2,3\rangle$. The dypyramid is divided into the upper and the lower (upside down) tetrahedrons. Correspondingly, the configurations (1-, 2-, 3 -walls) are constructed by joining two tetrahedral solutions at the $z=0$ plane.
The middle row of Fig. 7 corresponds to $a_{45}=0$ where the strengths of all the vacua are comparable. Namely, all the vacuum domains meet at the origin. Accordingly, the inner 2-wall corresponding to the triangle $\langle 1,2,3\rangle$ disappears.

As increasing $a_{45}$ further, the vacuum domains $\langle 4\rangle$ and $\langle 5\rangle$ expand and directly meet to create new 1 -wall $\langle 4,5\rangle$; see the third row of Fig. 7. In this case, the whole dypyramid can be thought of as the sum of three subtetrahedrons $\langle 1,2,4,5\rangle$, $\langle 1,3,4,5\rangle$, and $\langle 2,3,4,5\rangle$. This can be seen in the 2- and 3 -walls depicted in the third row of Fig. 7.

Finally, we consider a rare case that the four vacua are placed on a plane similarly to Fig. 6 . We now put the four points on a plane so that they form a square; see Fig. 8. Namely, the five vacua form a square pyramid. As an example, our choice is

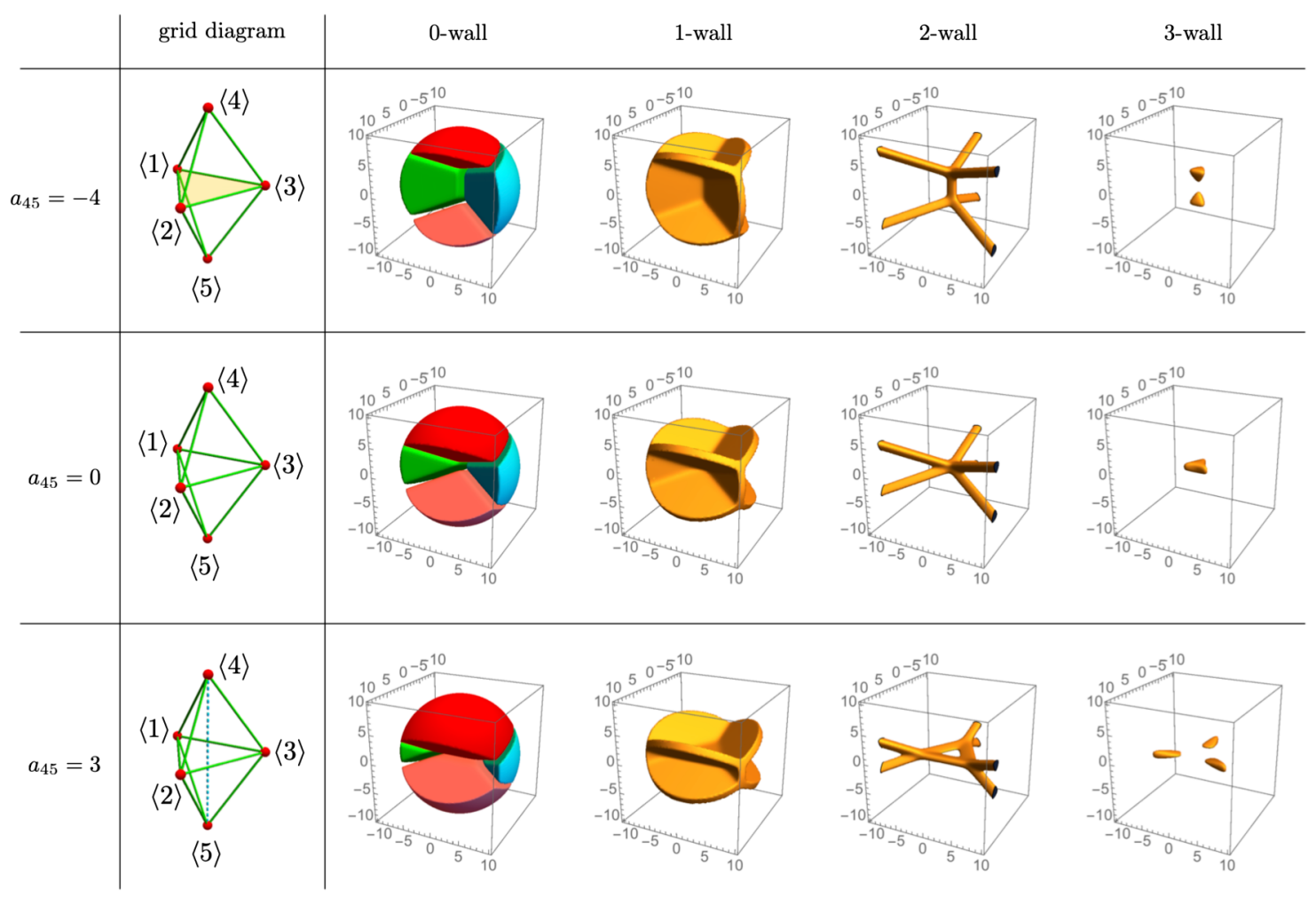

FIG. 7. Exact solutions in three typical branches for the grid diagram in Fig. 4(b). Isosurfaces of the topological charge densities $\mathcal{W}_{d}$ for $d$ walls $(d=0,1,2,3)$ are shown. 


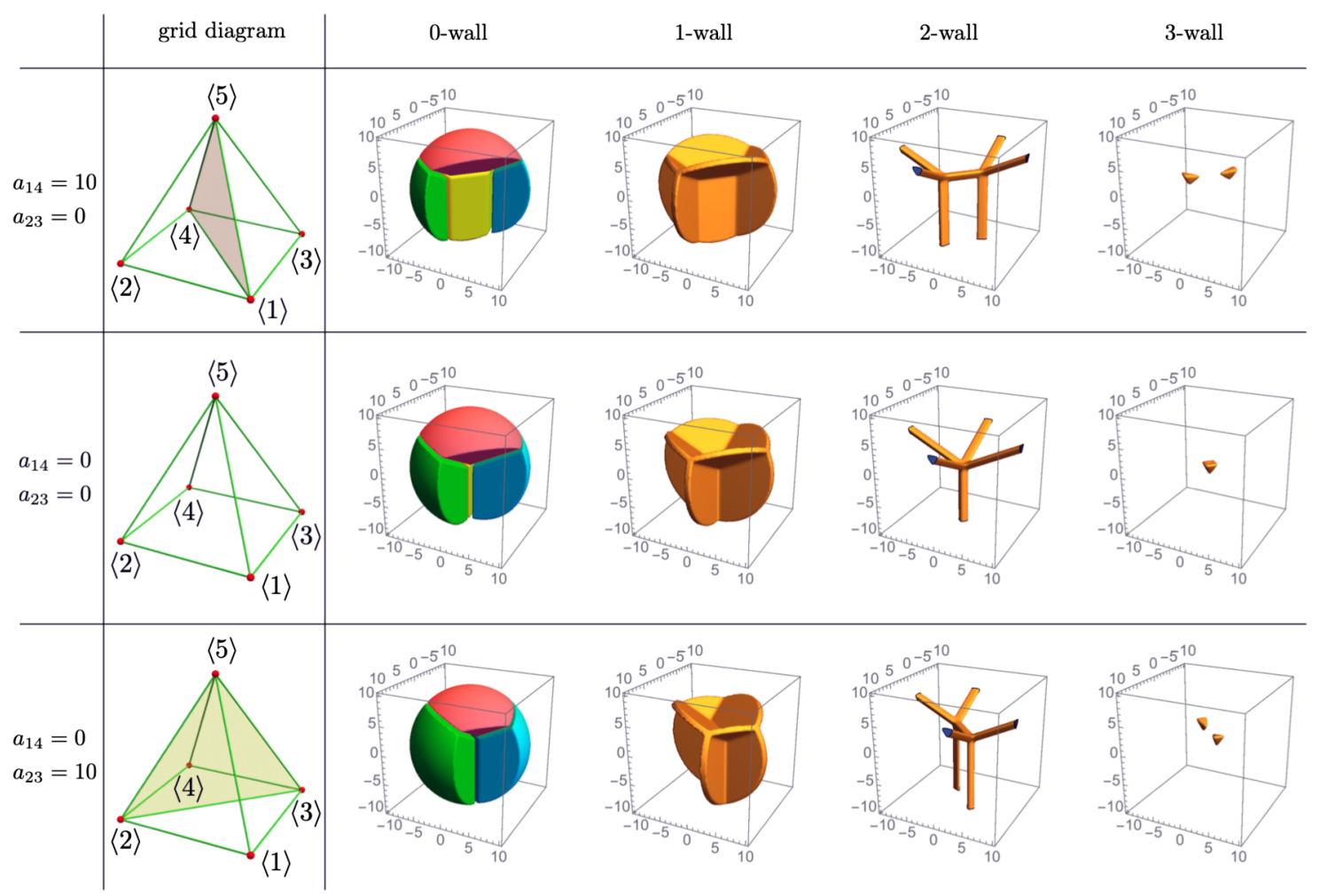

FIG. 8. Exact solutions in three typical branches for the fine-tuned grid diagram. Isosurfaces of the topological charge densities $\mathcal{W}_{d}$ for $d$ walls $(d=0,1,2,3)$ are shown.

$$
\begin{gathered}
\boldsymbol{m}_{\mathbf{1}}=(2,2,0), \\
\boldsymbol{m}_{\mathbf{2}}=(2,-2,0), \\
\boldsymbol{m}_{\mathbf{3}}=(-2,2,0), \\
\boldsymbol{m}_{\mathbf{4}}=(-2,-2,0), \\
\boldsymbol{m}_{\mathbf{5}}=(0,0,4) .
\end{gathered}
$$

The moduli matrix useful for this case is

$$
H_{0}=\left(e^{a_{14}}, e^{a_{23}}, e^{a_{23}}, e^{a_{14}}, 1\right),
$$

where $a_{14}$ corresponds to the weights of $\langle 1\rangle$ and $\langle 4\rangle$ whereas $a_{23}$ corresponds to the weights of $\langle 2\rangle$ and $\langle 3\rangle .^{2}$ We show three typical solutions with $\left(a_{14}, a_{23}\right)=(10,0)$, $(0,0),(0,10)$ in Fig. 8.

In the first row of Fig. 8 , the first solution with $\left(a_{14}, a_{23}\right)=$ $(10,0)$ is shown. Since the weights of $\langle 1\rangle$ and $\langle 4\rangle$ are greater than those of $\langle 2\rangle$ and $\langle 3\rangle$, the former domains stick out and directly meet to form the 1 -wall $\langle 1,4\rangle$.

When $\left(a_{14}, a_{23}\right)=(0,0)$, all the vacua have the same influences, so that they meet at a point; see the middle row of Fig. 8.

\footnotetext{
${ }^{2}$ The parameters $a_{14}$ and $a_{23}$ are not physically independent moduli due to the $V$ transformation (3.20).
}

When $\left(a_{14}, a_{23}\right)=(0,10)$, the influence of $\langle 2\rangle$ and $\langle 3\rangle$ is the maximum, so that their domains contact and form the 1-wall $\langle 2,3\rangle$, as shown in the third row of Fig. 8.
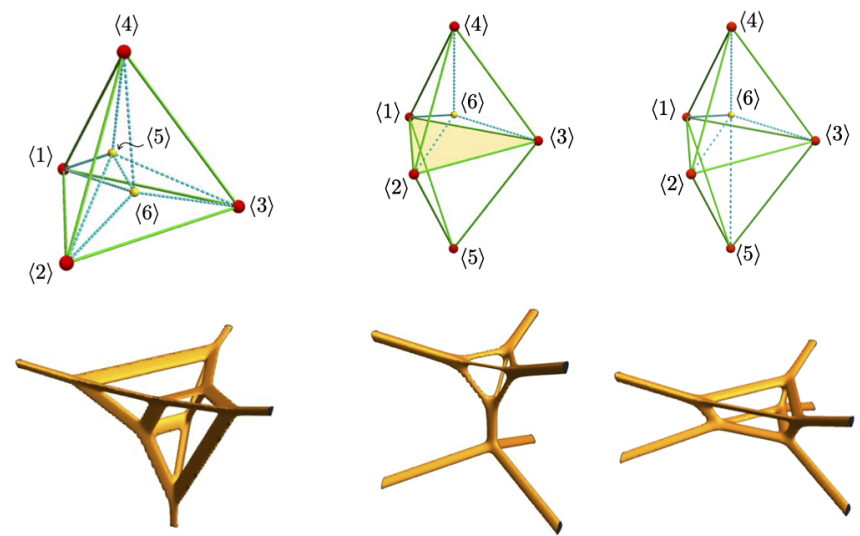

(a)

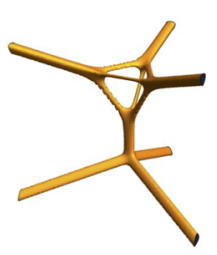

(b1)

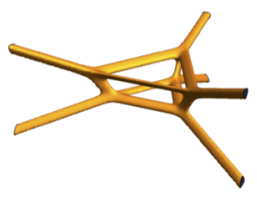

(b2)
FIG. 9. Exact solutions of domain wall networks for the model with $N=6$. The first line shows the grid diagrams, and the second line shows isosurfaces of $\mathcal{W}_{2}$ for the corresponding solutions. (a) The grid diagram is a tetrahedron and it has two inner vertices. (b1) and (b2) are two different type of the networks for the dipyramid-form grid diagram with one inner vertex. (a) has two vacuum bubbles, and (b1) and (b2) have one vacuum bubble. 

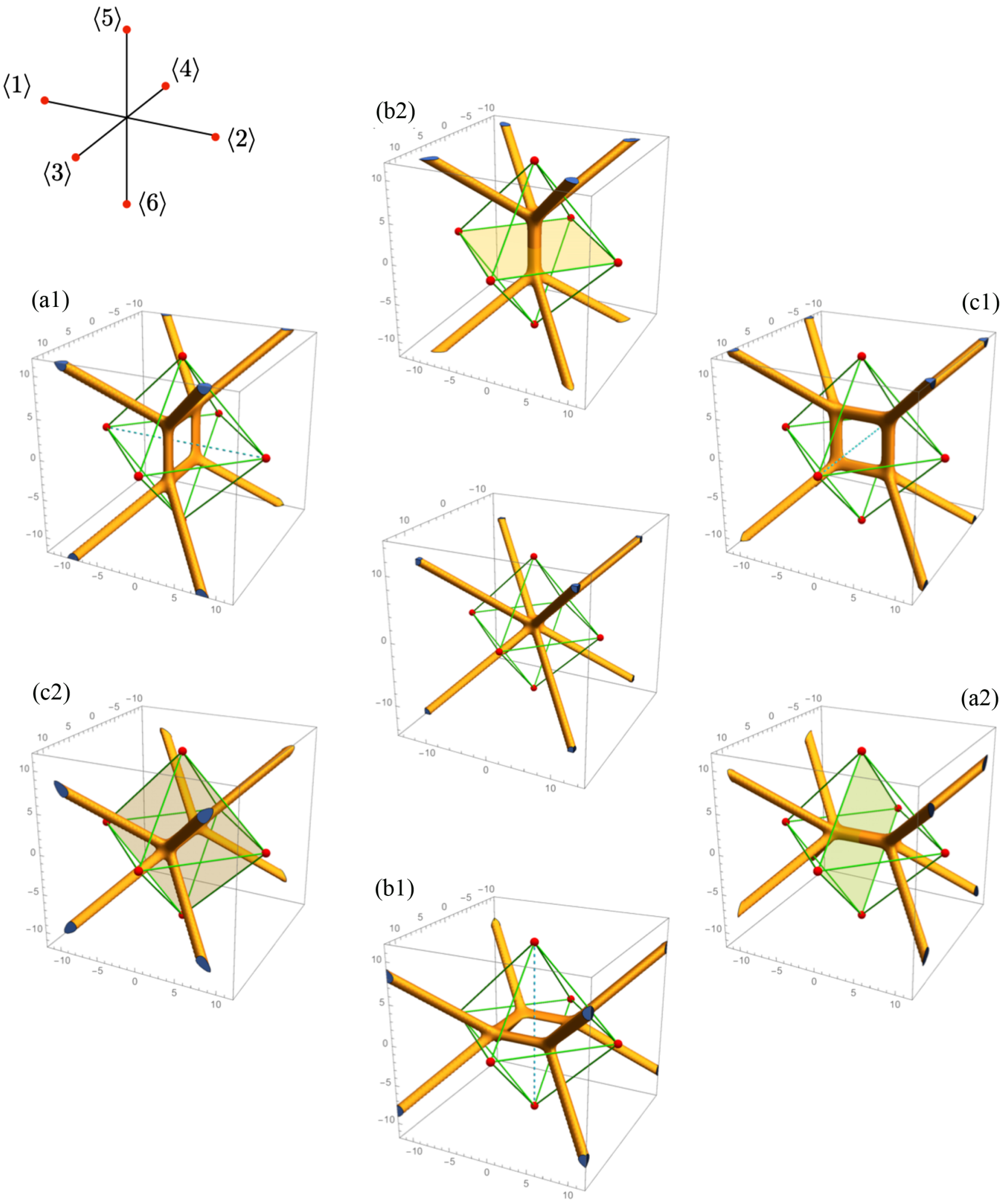

FIG. 10. Exact solutions of domain wall network in the octahedral grid diagram $(N=6)$ without inner vertices. We superpose the two kinds of graphs, the one is the $\mathcal{W}_{2}$ in the $\boldsymbol{x}$ space and the other is the grid diagram in the $\boldsymbol{\Sigma}$ space. There are three orthogonal directions to deform the network. The figure at the center shows the network where all the six vacua have the same influences. (a1) [(a2)] is obtained when the weight of $\langle 1\rangle$ and $\langle 2\rangle$ is larger (smaller) than the other vacua. Similarly, (b1) and (b2) [(c1) and (c2)] transit by controlling the relative influence of $\langle 5\rangle$ and $\langle 6\rangle[\langle 3\rangle$ and $\langle 4\rangle]$. 
A difference of the two cases $\left(a_{14}, a_{23}\right)=(10,0)$ and $(0,10)$ is just the difference of dividing the square pyramid into two tetrahedrons. If we only look at the square face, it is a transition between the $s$ and $t$ channels found for a planar 1-wall network, discussed in Ref. [75]. Therefore, this sort of cutting the square pyramid into two tetrahedrons is a three-dimensional analog of the transition between the $s$ and $t$ channels in two dimensions. One can be convinced by looking at the 2-wall (but not 1-wall) configurations in Fig. 8 from above (or bottom).

\section{D. $N=6$ : Octahedron}

Let us further increase the number of vacua, namely, $N=6$. There are six vacua. There are three different cases according to the convex polyhedra made by connecting the vacua. The first case is a tetrahedron with two inner vertices, the second is a dipyramid with a inner vertex, and the third is a octahedron without inner vertices.

The tetrahedral case is shown in Fig. 9(a). The whole tetrahedron is divided into eight subtetrahedrons $\langle 1,2,3,6\rangle$, $\langle 1,2,4,5\rangle,\langle 1,3,4,5\rangle,\langle 2,3,4,6\rangle,\langle 1,2,5,6\rangle,\langle 2,3,5,6\rangle$, $\langle 3,4,5,6\rangle,\langle 1,4,5,6\rangle$. Correspondingly, there exist eight 3 -walls (junctions of four 2-walls). The two inner vertices give rise to the two vacuum bubbles as shown in the bottom figure of Fig. 9(a). The concrete moduli matrix for Fig. 9(a) is

$$
H_{0}=\left(1,1,1,1, e^{a_{5}}, e^{a_{6}}\right)
$$

with $a_{5}=a_{6}=10$. The moduli parameter $a_{5}\left(a_{6}\right)$ controls the size of the bubble of $\langle 5\rangle(\langle 6\rangle)$.

For the dipyramid type case, there are two branches according to how to divide it into subtetrahedrons. The first branch is shown in Fig. 9(b1) in which the whole dipyramid is divided into five tetrahedrons, $\langle 1,2,3,6\rangle,\langle 1,2,4,6\rangle$, $\langle 1,3,4,6\rangle,\langle 2,3,4,6\rangle$, and $\langle 1,2,3,5\rangle$. It can be also regarded as the octahedron made of two tetrahedrons bonded at the surface $\langle 1,2,3\rangle$. The upper tetrahedron has the inner vertex, whereas the bottom one has no inner vertices. The resulting 2-wall wire frame shown in the lower figure of Fig. 9(b1) can be indeed obtained by connecting the 2-walls of Fig. 2 and the third row of Fig. 5.

On the other hand, the whole dipyramid is divided into six subtetrahedrons $\langle 1,2,4,6\rangle,\langle 2,3,4,6\rangle,\langle 1,3,4,6\rangle$, $\langle 1,2,5,6\rangle,\langle 2,3,5,6\rangle$, and $\langle 1,3,5,6\rangle$ in the second branch; see Fig. 9(b2).

The useful moduli matrix for describing this transition turns out to be

$$
H_{0}=\left(e^{a_{123}}, e^{a_{123}}, e^{a_{123}}, 1,1, e^{a_{6}}\right) .
$$

The moduli parameter $a_{123}$ controls strength of the vacua $\langle 1\rangle,\langle 2\rangle$, and $\langle 3\rangle$. In other words, it is related to distance between the 3-walls $\langle 1,2,3,5\rangle$ and $\langle 1,2,3,6\rangle$. The other $a_{6}$ controls the size of the bubble $\langle 6\rangle$. We choose $\left(a_{123}, a_{6}\right)=$ $(10,10)$ in Fig. 9(b1) and $\left(a_{123}, a_{6}\right)=(-2,4)$ in Fig. 9(b2).

Finally, we show the octahedral case in Fig. 10. For simplicity, we set the eight masses $\boldsymbol{m}_{A}$ at vertices of a regular octahedron. This configuration can be understood as follows. To understand this configuration, we arrange the six vacua to the following three pairs: $\langle 1\rangle-\langle 2\rangle,\langle 3\rangle-\langle 4\rangle$, and $\langle 5\rangle-\langle 6\rangle$. Then, the appropriate moduli matrix is

$$
H_{0}=\left(e^{a_{12}}, e^{a_{12}}, e^{a_{34}}, e^{a_{34}}, e^{a_{56}}, e^{a_{56}}\right),
$$

where only two among $a_{12}, a_{34}, a_{56}$ are independent. When the weight of the vacua $\langle 1\rangle$ and $\langle 2\rangle$ are larger than the other vacua, the vacua $\langle 1\rangle$ and $\langle 2\rangle$ directly meet to form the 1-wall $\langle 1,2\rangle$. It corresponds to (a1) of Fig. 10 in which the vertices $\langle 1\rangle$ and $\langle 2\rangle$ are connected by the dashed segment, and at the same time there is an inner loop of the 2 -wall penetrated by the segment. As the weight relatively decreases, the loop shrinks. When $a_{12}=a_{34}=a_{56}$, all the vacua are equivalent and there are no 2-wall loops, as shown in the center panel of Fig. 10. If we further reduce $a_{12}$ as $a_{12}<a_{34}=a_{56}$, the four vacua $\langle 3\rangle,\langle 4\rangle,\langle 5\rangle$, and $\langle 6\rangle$ stick out and directly meet each other; see Fig. 10(a2). The same can be said to the other pairs $\langle 3\rangle-\langle 4\rangle$ and $\langle 5\rangle-\langle 6\rangle$. When $a_{56}>a_{12}=a_{34}\left(a_{56}<a_{12}=a_{34}\right)$, we have (b1) [(b2)] of Fig. 10. When $a_{34}>a_{12}=a_{56}\left(a_{34}<a_{12}=a_{56}\right)$, we have (c1) [(c2)] of Fig. 10. We can rephrase these as follows. The configurations of (a1), (b1), and (c1) correspond to the decomposition of the octahedron into four subtetrahedrons. On the other hand, (a2), (b2), and (c2) correspond to the decomposition of the octahedron into two square pyramids.

\section{PLATONIC, ARCHIMEDEAN, CATALAN, AND KEPLER-POINSOT VACUUM BUBBLES}

In the previous sections, we have seen several examples in which the configurations have vacuum bubbles; see Figs. 5 and 9. They can appear when the grid diagrams which are generally convex polytopes have inner vertices. The number of the inner vertices is equal to the number of the vacuum bubbles. The size of a vacuum bubble is controlled by a modulus. The bubble is sometimes invisibly small, and we need to take the corresponding moduli parameter sufficiently large to broaden the bubble.

\section{A. The Platonic vacuum bubbles}

Here, we are interested in the shape of the vacuum bubble. In Fig. 5, we met the bubble which is a regular tetrahedron. There are two conditions for a regular tetrahedral bubble to exist. One is that the grid diagram is a regular tetrahedron, and the other is that the inner vertex is located at the center of the regular tetrahedron. If we relax either or both conditions, the bubble deforms accordingly. 


\section{Platonic solids}
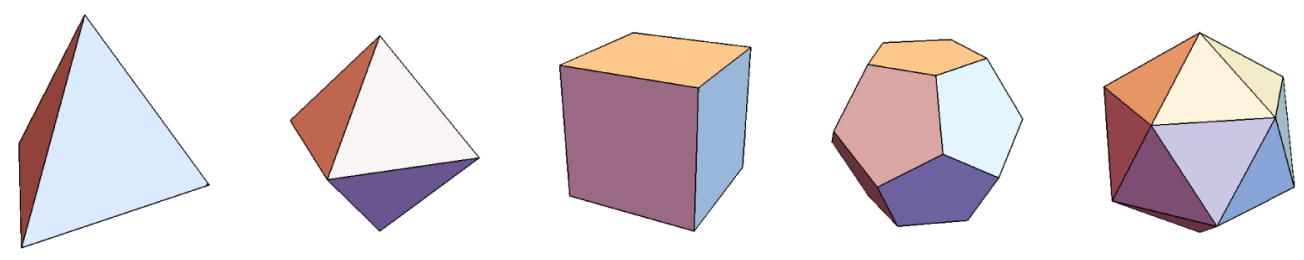

\section{Platonic bubbles}
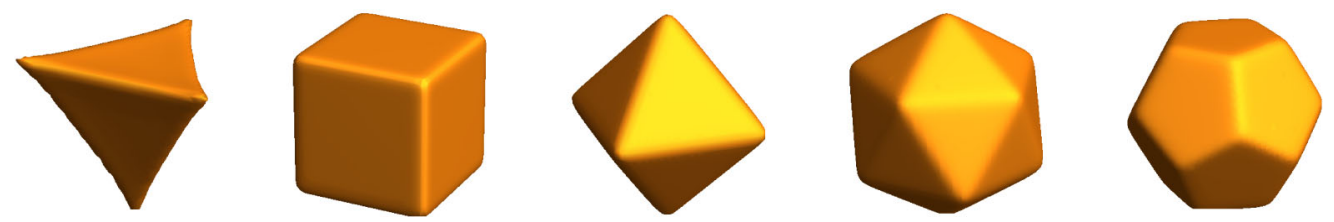

\section{Platonic wire frames}
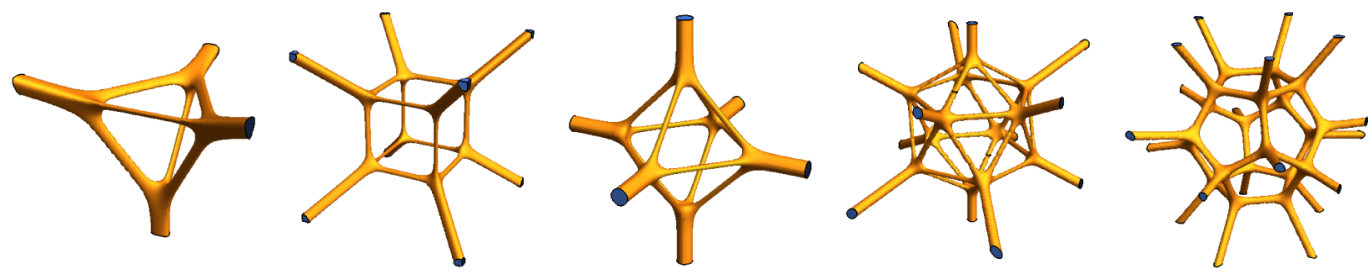

FIG. 11. The top row shows five grid diagrams which are congruent with the Platonic solids. The inner vertices at the center are hidden. The second row shows the vacuum bubbles for each grid diagrams, and the third row shows the 2-wall charge densities $\mathcal{W}_{2}$. The bubble shape and the grid diagram are dual each other.

Now, one notices that the two regular tetrahedrons in the grid diagrams and in the real space in Fig. 5 are upside down. This should be so, since each face of the bubble is perpendicular to the inner edge of the grid diagram. This can be rephrased as follows. The shape of the vacuum bubble is equivalent to a shape obtained by exchanging the outer vertices and faces of the grid diagram. Such a polyhedron is called dual of the original polyhedron. Since the regular tetrahedron is known to be self-dual, the grid diagram and the vacuum bubble are both regular tetrahedrons. We show the regular tetrahedron as the grid diagram, the dual tetrahedron as the vacuum bubble, and an isosurface of the 2-wall density in the leftmost column in Fig. 11.

To understand the duality better, let us next consider domain wall network in a shape of the other convex regular polyhedra: octahedron, cube, icosahedron, and dodecahedron. Namely, we consider the grid diagrams which are congruent with the Platonic solids and put an inner vertex at their centers.
For example, we consider $N=6+1$ for the octahedron with the inner vertex. Let the first six flavors $(A=1,2$, $\ldots, 6)$ correspond to the vertices of the octahedron and the seventh flavor $(A=7)$ to the inner vertex. To get a vacuum bubble of a desired shape, we consider the moduli matrix $H_{0}=\left(1,1,1,1,1,1, e^{a_{7}}\right)$. Then we take a sufficiently large value for $e^{a_{7}}$ to broaden the vacuum bubble $\langle 7\rangle$. The result is shown in the second column from the left of Fig. 11. We indeed observe that the resultant vacuum bubble takes a shape of a regular cube, dual to a regular octahedron.

Similarly, we take $N=8+1$ for a regular cube plus an inner vertex, $N=20+1$ for a regular dodecahedron plus an inner vertex, and $N=12+1$ for a regular icosahedron plus an inner vertex. We find that the shapes of the vacuum bubbles are a regular octahedron, icosahedron, and dodecahedron for the regular cube, dodecahedron, and icosahedron, respectively; see Fig. 11. Thus, putting an inner vertex at the centers of the Platonic solids and broadening the corresponding vacuum give us the regular 
Archimedean solids
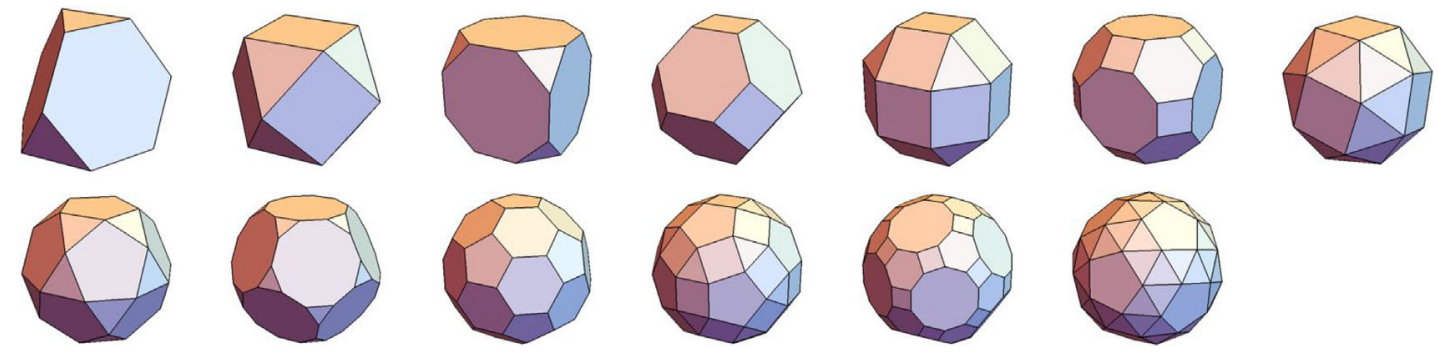

\section{Catalan bubbles}
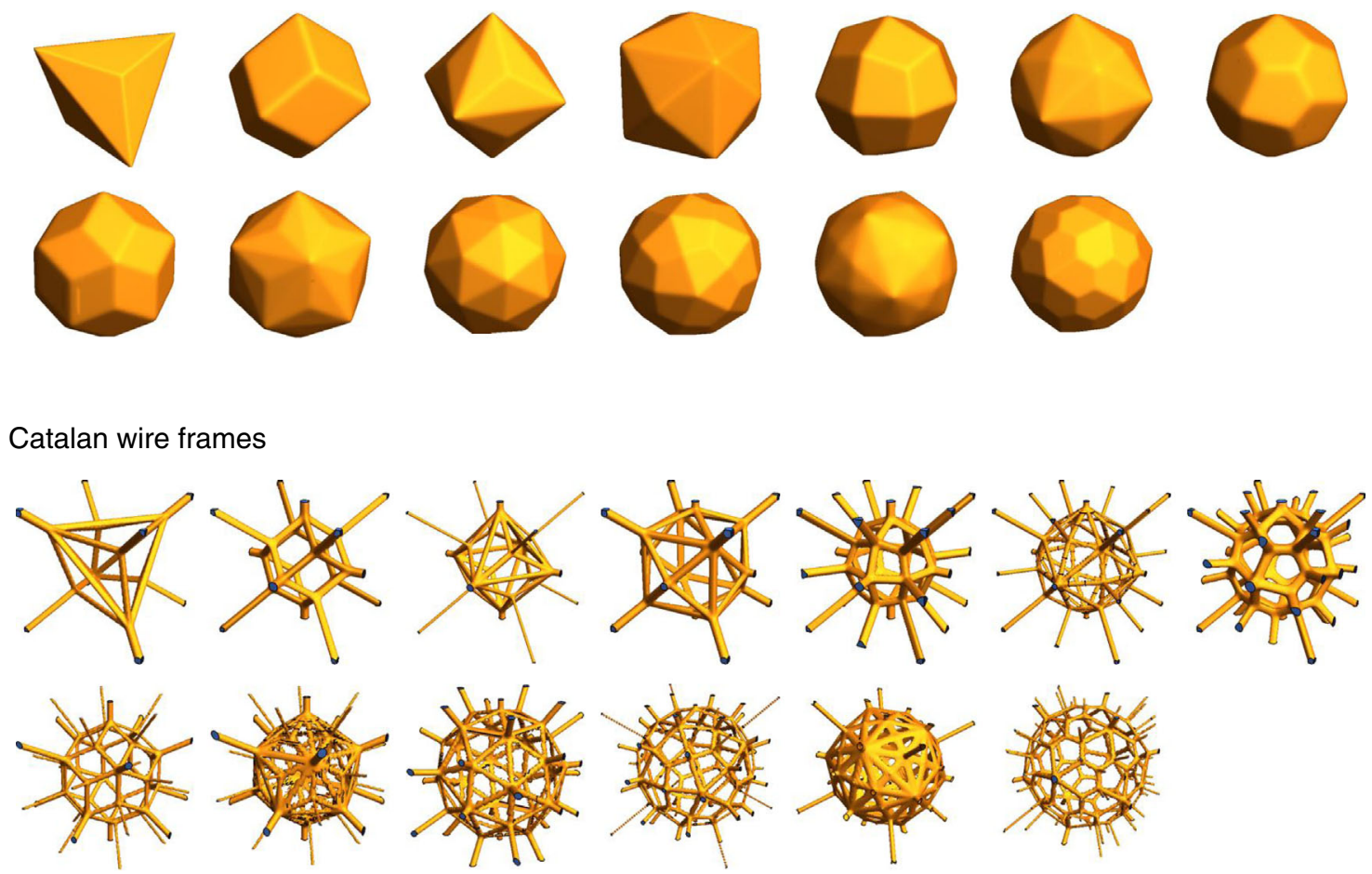

FIG. 12. The top row shows 13 grid diagrams which are congruent with the Archimedean solids. The inner vertices at the center are hidden. The second row shows the vacuum bubbles for each grid diagrams, and the third row shows the 2-wall charge densities $\mathcal{W}_{2}$. The bubbles are dual to the grid diagram, so that their shapes are congruent with 13 Catalan solids.

bubble polyhedra dual to the Platonic solids as the grid diagrams.

Here, we equally set all the weights of the outer vertices to be 1 to have the regular polyhedrons. Of course, if we choose a generic moduli matrix, the vacuum bubbles deform accordingly, as mentioned at the beginning of this subsection.

\section{B. The Archimedean and Catalan vacuum bubbles}

Let us also test the idea to the other classic polyhedra, 13 Archimedean polyhedra and their duals called the Catalan polyhedra. The Archimedean polyhedra are uniform polyhedra which are vertex transitive. Therefore, the Catalan polyhedra are face transitive.
Figure 12 shows the vacuum bubbles and the 2-wall charge densities for the Archimedean grid diagrams with an additional inner vertex at the center. As desired, the bubble shapes are dual to the grid diagrams, namely, we indeed have the Catalan bubbles. Let us explain how to construct these bubbles by taking the truncated tetrahedron (the solids at the left-top corners in Fig. 12) as an example. We need $N=12+1$ flavors and put 12 masses on 12 vertices of the truncated tetrahedron. In addition, we put the thirteenth mass at the center of the truncated tetrahedron. Then we take the moduli matrix $H_{0}=\left(1,1, \ldots, 1, e^{a_{13}}\right)$ with $e^{a_{13}}$ being sufficiently large. This way, we get the triakis tetrahedron as the vacuum bubble, dual to the truncated tetrahedron. All the rest solids can be obtained by similar procedures. 
Catalan solids
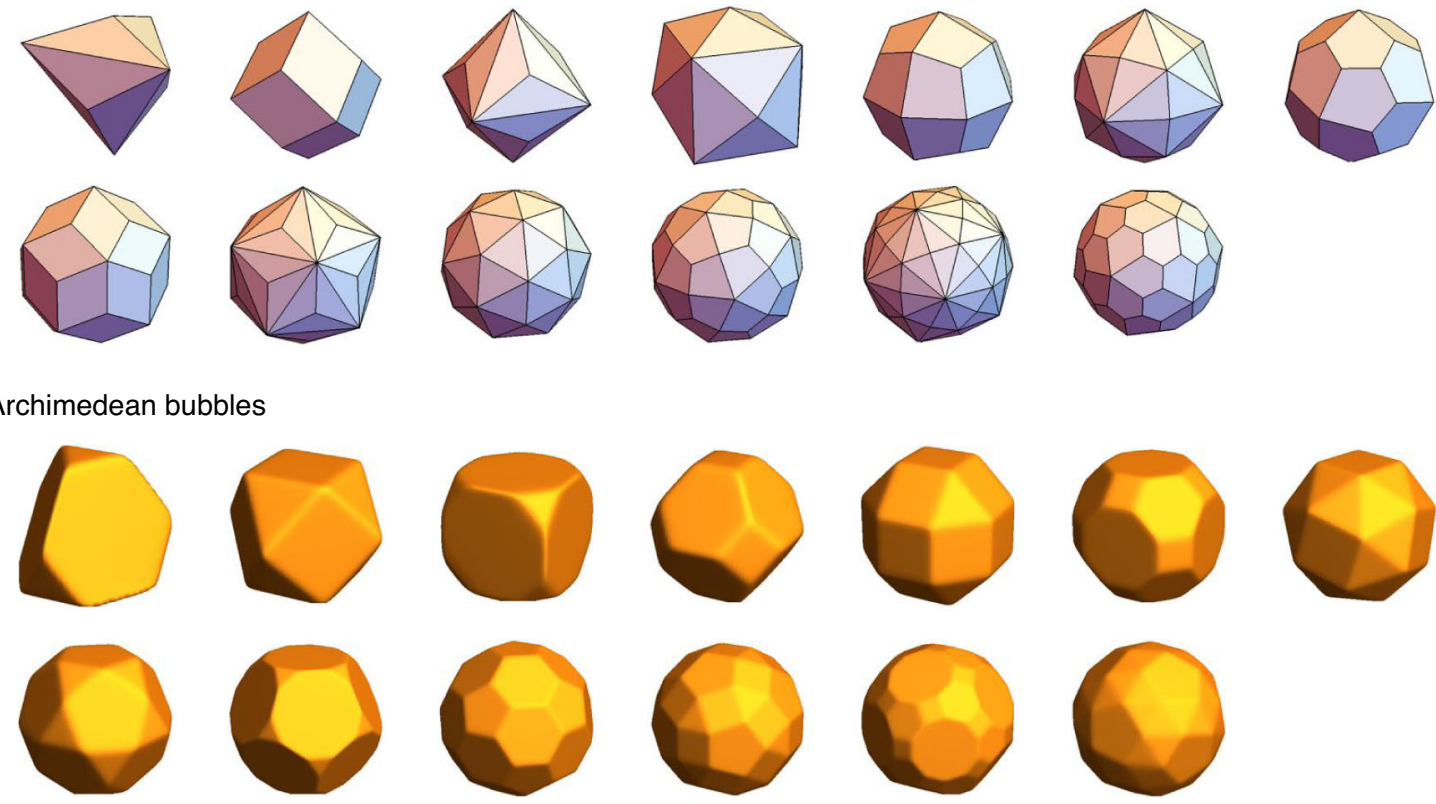

Archimedean wire frames
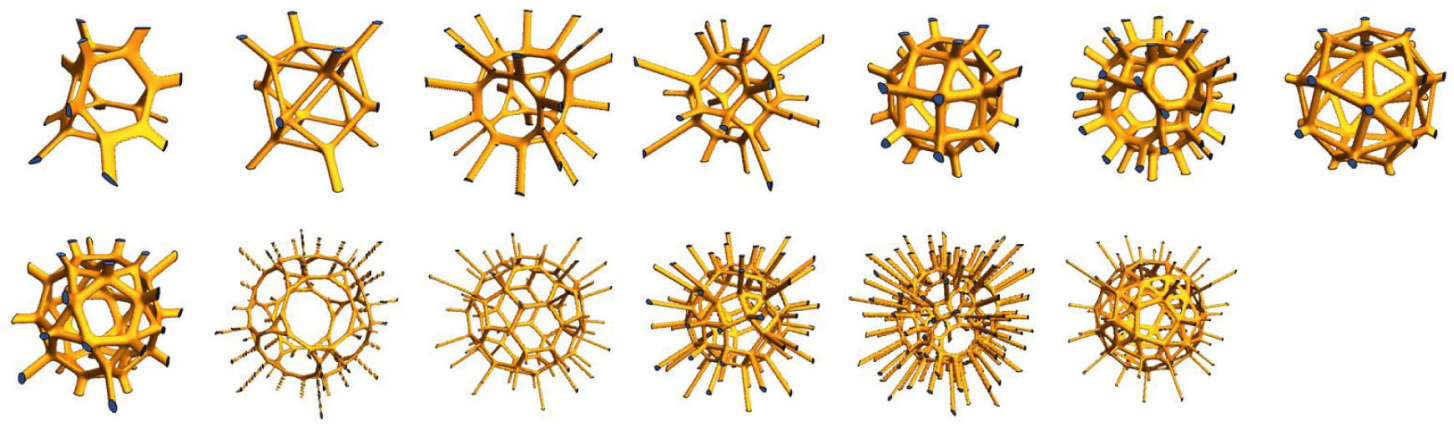

FIG. 13. The top row shows 13 grid diagrams which are congruent with the Catalan solids. The inner vertices at the center are hidden. The second row shows the vacuum bubbles for each grid diagrams, and the third row shows the 2-wall charge densities $\mathcal{W}_{2}$. The bubbles are dual to the grid diagram, so that their shapes are congruent with 13 Archimedean solids.

Figure 13 shows the vacuum bubbles and the 2-wall charge densities for the Catalan grid diagrams with an additional inner vertex at the center. As expected, the bubble shapes are dual to the grid diagrams, namely, we have the Archimedean bubbles. Let us explain how to construct these bubbles by taking the triakis tetrahedron (the solids at the left-top corners in Fig. 13) as an example. We need $N=$ $8+1$ flavors and put eight masses on eight vertices of the trikias tetrahedron. In addition, we put the ninth mass at the center of the trikias tetrahedron. Then we take the moduli matrix $H_{0}=\left(1,1, \ldots, 1, e^{a_{9}}\right)$ with $e^{a_{9}}$ being sufficiently large. This way, we get the truncated tetrahedron as the vacuum bubble, dual to the trikias tetrahedron. All the rest solids can be obtained by similar procedures.

\section{The Kepler-Poinsot vacuum bubbles}

So far, we have only studied the convex polyhedra. Before closing this section, let us briefly mention star polyhedra. Here, we take the small and great stellated dodecahedron. Namely, we consider the grid diagrams which are congruent with the star polyhedra. Stellating a dodecahedron to a stellated dodecahedron is a threedimensional analog of stellating a pentagon to a pentagram.

In order to obtain an intuitive picture, let us first consider two-dimensional domain wall network for a grid diagram which is congruent with a pentagram. The ten vacua are located on vertices of the pentagram as shown in Fig. 14. The pentagram consists of large (the red five vertices) and small (the green five vertices) pentagons. The five vacua corresponding to the small pentagon lead to five vacuum bubbles. When we equally broaden them by the moduli matrix $H_{0}=\left(1,1,1,1,1, e^{a}, e^{a}, e^{a}, e^{a}, e^{a}\right)$ with sufficiently large $e^{a}$, the bubbles $(\langle 6\rangle,\langle 7\rangle,\langle 8\rangle,\langle 9\rangle,\langle 10\rangle)$ form a stellated pentagon as shown in Fig. 14.

We are ready to study three-dimensional domain wall networks with the grid diagrams congruent with the small 


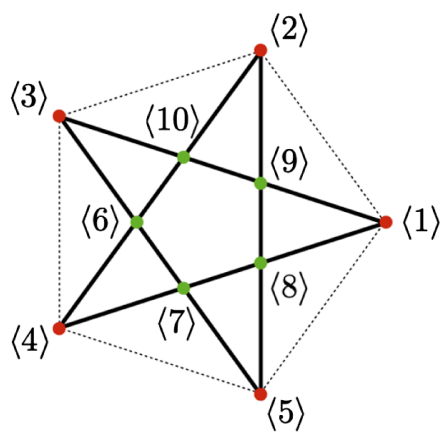

grid diagram

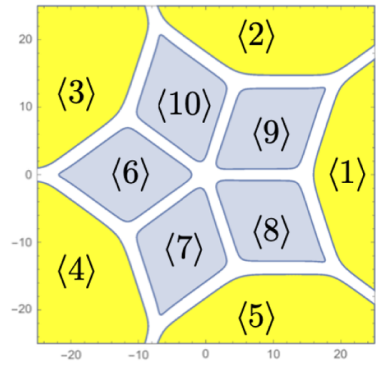

vacuum bubble
FIG. 14. The left panel shows the grid diagram congruent with a pentagram. The right panel shows an exact solution with five vacuum bubbles which have the same influence. The bubbles form a stellated pentagon.

and great stellated dodecahedron shown in Fig. 15. The former consists of a large icosahedron with 12 red vertices and a small dodecahedron with 20 green vertices. The vertices of the small dodecahedron are inner vacua. Therefore, by broadening them with the equal weight, we have 20 vacuum bubbles which form the great stellated dodecahedron as shown in the left-bottom panel of Fig. 15. On the other hand, the latter is made of the large dodecahedron with the green vertices and the small icosahedron with the red vertices. Now, the vertices of the dodecahedron become the inner vacua. So, when we

\section{Kepler-Poinsot solids}

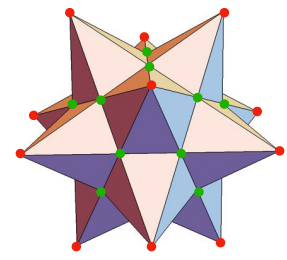

small stellated dodecahedron

Kepler-Poinsot bubbles

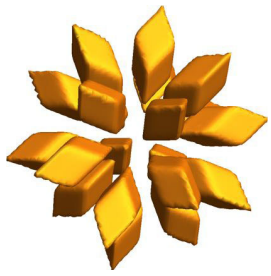

great stellated dodecahedron

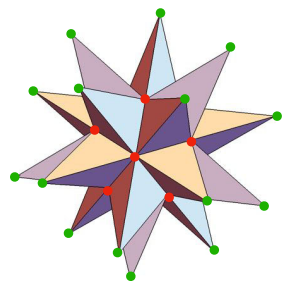

great stellated dodecahedron

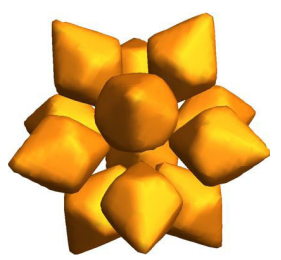

small stellated dodecahedron
FIG. 15. The top row: the two regular star solids categorized into the Keplar-Poinsot solids are used as the grid diagrams. The bottom row: the corresponding vacuum bubbles. The small (great) stellated dodecahedron yields the great (small) stellated dodecahedral bubble. equally broaden them, we have 12 vacuum bubbles which form the small stellated dodecahedron as shown in the right-bottom panel of Fig. 15. In short, when the grid diagram is the small stellated dodecahedron, the vacuum bubbles form the great stellated dodecahedron, and vice versa. Although the small and great stellated dodecahedrons are not dual to each other (dual of the small stellated dodecahedron is the great dodecahedron while that of the great stellated dodecahedron is the great icosahedron), here, we found they exchange via the vacuum bubbles.

\section{SUMMARY AND DISCUSSION}

In this paper, we have proceeded to the study on the nonplanar BPS domain wall junctions in the Abelian gauge theory with the $N$ Higgs fields $H_{A}$ and the $D$ neutral scalar fields $\Sigma_{m}$ in $D+1$ dimensions, which were recently proposed in Ref. [86]. In the previous work [86], we obtained the new exact BPS solutions of the single domain wall junctions associated with a particular symmetry breaking pattern $\mathcal{S}_{D+1} \rightarrow \mathcal{S}_{D}\left(\mathcal{S}_{D}\right.$ is the symmetric group of rank $D$ ) in $D+1$ dimensions. We also needed to impose the symmetry $\mathcal{S}_{D+1}$ in addition to the special relation between the model parameters (the masses, the gauge coupling, and the Fayet-Illiopoulos term) for having the exact solutions in [86].

Generalizing the previous study, the present work has dealt with the generic network of domain walls including multiple junctions. We have not imposed any particular discrete symmetry in this paper. We have succeeded in solving partially the BPS equations by the moduli matrix formalism and finding all the moduli parameters of the generic domain wall network solutions. While the master equation (3.19) cannot be analytically solved in general, we focused on the infinite $U(1)$ gauge coupling limit in which the model reduces to the $\mathbb{C} P^{N-1}$ model and the BPS equations including the master equation are fully solvable for any moduli parameters. These are the first analytic solutions for nonplanar domain wall networks in $D$ dimensions $(D \geq 3)$.

As demonstrated on showing how the nonplanar networks look like, we have studied the $\mathbb{C} P^{N-1}$ model in $D=$ 3 for $N=4,5,6$ in detail. In the case of $N=4$, the solution has only one junction. The solution is similar to one found in [86], but is more generic. The solutions in this paper can be obtained for any grid diagrams congruent with tetrahedra in contrast to the previous case [86] in which the grid diagram was limited to the regular tetrahedron. The network structure appears for $N>4$. For $N=5$, we have found two different types of networks in general. The first type has a vacuum bubble (a compact domain) surrounded by the four semi-infinite vacuum domains. The other type does not have any bubbles but all the five vacuum domains are semi-infinitely extended. The corresponding grid diagram is the tetrahedron with an inner vertex for the former 
and the dipyramid for the latter. We have shown the network shape is controlled by one moduli parameter. We also have studied the special cases in which the four vertices are on a plane while the fifth vertex is off the plane. In the $N=6$ case, there are three different types in general. With respect to the grid diagram, they correspond to the tetrahedron with two inner vertices, the dipyramid with one inner vertex, and the octahedron without any inner vertices. Accordingly, there appear two, one, and no vacuum bubbles in the networks, respectively. As in the case of $N=5$, we have explicitly shown how the network shape changes according with the moduli parameters. They are essentially controlled by the two moduli besides the translations, either the size of the bubble or the distance between junctions.

Finally, we have constructed the beautiful polyhedra known from ancient times as domain wall networks. We have started with the grid diagrams congruent with five Platonic solids with an inner vertex at the centers. The network solutions have single vacuum bubbles corresponding to the inner vertices, and we have found that shapes of the bubble are dual to the grid diagrams. In addition to the regular polyhedra, we have also investigated the semiregular polyhedra, the Archimedean solids. The corresponding vacuum bubbles are again dual to the Archimedean, namely, the Catalan solids. Conversely, the grid diagrams congruent with the Catalan solids lead to the bubbles congruent with the Archimedean solids. Our final examples have been star polyhedra. We have taken two well-known star polyhedra from the Kepler-Poinsot solids, the small and great stellated dodecahedrons. The former (latter) has 20 (twelve) inner vertices. Accordingly, the same number of the bubbles appears in the networks. Interestingly, the bubbles in the former case form the great stellated dodecahedron and those in the latter case form the small stellated dodecahedron.

Before closing this work, let us make several comments on future directions. First, although we have established the generic formulae for the exact solutions of the domain wall networks in the limit of the $\mathbb{C} P^{N-1}$ model in generic $D+1$ dimensions, we only have shown concrete configurations in the $D=3$ case. For $D \geq 4$, the networks become more complicated and their deformations by changing moduli parameters are intricate. We will explain such higher- dimensional domain wall networks in more details elsewhere.

Second, we only have studied the Abelian gauge theories and the massive $\mathbb{C} P^{N-1}$ model as the infinite gauge coupling limit in this paper. The non-Abelian generalization was obtained in $D=2[75,76]$. We will study non-Abelian nonplanar domain wall networks in higher dimensions $D \geq 3$ elsewhere.

Third, we have met the polyhedra and well-known mathematical notions like the duality in study of the domain walls. While the mathematical solids are sharp objects, the vacuum bubbles found in this work are rounded off. We expect that the domain wall networks would mathematically be useful for studying such melting polyhedra and polytopes as the case of Amoeba and tropical geometry discussed in $D=2$ [80].

Fourth, the grid diagrams in the $\boldsymbol{\Sigma}$ space and the networks in $\boldsymbol{x}$ space seem to be very similar to the Delaunay diagram and the Voronoi diagram appearing in vast area of Science.

We expect our exact solutions of the $D$-dimensional domain wall networks and the grid diagrams would be applicable to many areas. Josephson junctions of superconductors $[87,88]$ are one of interesting applications.

\section{ACKNOWLEDGMENTS}

This work was supported by the Ministry of Education, Culture, Sports, Science (MEXT)-Supported Program for the Strategic Research Foundation at Private Universities "Topological Science" (Grant No. S1511006) and JSPS KAKENHI Grant No. 16H03984. The work of M. E. is also supported in part by JSPS Grant-in-Aid for Scientific Research KAKENHI Grant No. JP19K03839 and by MEXT KAKENHI Grant-in-Aid for Scientific Research on Innovative Areas "Discrete Geometric Analysis for Materials Design" Grant No. JP17H06462 from the MEXT of Japan. The work of M. N. is also supported in part by JSPS KAKENHI Grant No. $18 \mathrm{H} 01217$ by a Grantin-Aid for Scientific Research on Innovative Areas "Topological Materials Science" (KAKENHI Grant No. 15H05855) from MEXT of Japan.
[1] N. S. Manton and P. Sutcliffe, Topological Solitons, Cambridge Monographs on Mathematical Physics (Cambridge University Press, Cambridge, United Kingdom, 2004).

[2] T. Vachaspati, Kinks and Domain Walls: An Introduction to Classical and Quantum Solitons (Cambridge University Press, Cambridge, United Kingdom, 2006).
[3] R. Rajaraman, Solitons and Instantons. An Introduction to Solitons and Instantons in Quantum Field Theory (North-holland, Amsterdam, The Netherlands, 1982), p. 409

[4] T. W. B. Kibble, Some implications of a cosmological phase transition, Phys. Rep. 67, 183 (1980).

[5] T. W. B. Kibble, Topology of cosmic domains and strings, J. Phys. A 9, 1387 (1976). 
[6] W.H. Zurek, Cosmological experiments in superfluid helium? Nature (London) 317, 505 (1985).

[7] W. H. Zurek, Cosmological experiments in condensed matter systems, Phys. Rep. 276, 177 (1996).

[8] F. B. Humphrey, Domain walls and wall structure, in Science and Technology of Nanostructured Magnetic Materials, edited by G. C. Hadjipanayis and G. A. Prinz, NATO ASI Series (Series B: Physics) Vol. 259 (Springer, Boston, MA, 1991).

[9] L. Yin, H. Jiang, J. Qiao, and L. He, Direct imaging of topological edge states at a bilayer graphene domain wall, Nat. Commun. 7, 11760 (2016).

[10] I. Serban, B. Béri, A. R. Akhmerov, and C. W. J. Beenakker, Domain Wall in a Chiral p-Wave Superconductor: A Pathway for Electrical Current, Phys. Rev. Lett. 104, 147001 (2010).

[11] H. Takeuchi, K. Kasamatsu, M. Tsubota, and M. Nitta, Tachyon Condensation Due to Domain-Wall Annihilation in Bose-Einstein Condensates, Phys. Rev. Lett. 109, 245301 (2012).

[12] G. E. Volovik, The Universe in a Helium Droplet (Clarendon, Oxford, 2003).

[13] M. Eto, K. Hashimoto, and T. Hatsuda, Ferromagnetic neutron stars: Axial anomaly, dense neutron matter, and pionic wall, Phys. Rev. D 88, 081701 (2013).

[14] S. Yasui and M. Nitta, Domain walls in neutron ${ }^{3} P_{2}$ superfluids in neutron stars, Phys. Rev. C 101, 015207 (2020).

[15] M. Eto, Y. Hirono, M. Nitta, and S. Yasui, Vortices and other topological solitons in dense quark matter, Prog. Theor. Exp. Phys. 2014, 12D01 (2014).

[16] A. Vilenkin and E. P. S. Shellard, Cosmic Strings and Other Topological Defects (Cambridge University Press, Cambridge, United Kingdom, 2000).

[17] M. Bucher and D. N. Spergel, Is the dark matter a solid? Phys. Rev. D 60, 043505 (1999).

[18] E. B. Bogomolny, Stability of classical solutions, Yad. Fiz. 24, 861 (1976) [Sov. J. Nucl. Phys. 24, 449 (1976)].

[19] M. K. Prasad and C. M. Sommerfield, An Exact Classical Solution for the 't Hooft Monopole and the Julia-Zee Dyon, Phys. Rev. Lett. 35, 760 (1975).

[20] G. R. Dvali and M. A. Shifman, Domain walls in strongly coupled theories, Phys. Lett. B 396, 64 (1997); Erratum, Phys. Lett. B 407, 452 (1997).

[21] G. R. Dvali and M. A. Shifman, Dynamical compactification as a mechanism of spontaneous supersymmetry breaking, Nucl. Phys. B504, 127 (1997).

[22] A. Kovner, M. A. Shifman, and A. V. Smilga, Domain walls in supersymmetric Yang-Mills theories, Phys. Rev. D 56, 7978 (1997).

[23] A. V. Smilga and A. Veselov, Complex BPS Domain Walls and Phase Transition in Mass in Supersymmetric QCD, Phys. Rev. Lett. 79, 4529 (1997).

[24] B. Chibisov and M. A. Shifman, BPS saturated walls in supersymmetric theories, Phys. Rev. D 56, 7990 (1997); Erratum, Phys. Rev. D 58, 109901 (1998).

[25] V. S. Kaplunovsky, J. Sonnenschein, and S. Yankielowicz, Domain walls in supersymmetric Yang-Mills theories, Nucl. Phys. B552, 209 (1999).
[26] B. de Carlos and J. M. Moreno, Domain Walls in Supersymmetric QCD: From Weak to Strong Coupling, Phys. Rev. Lett. 83, 2120 (1999).

[27] G. R. Dvali, G. Gabadadze, and Z. Kakushadze, BPS domain walls in large $N$ supersymmetric QCD, Nucl. Phys. B562, 158 (1999).

[28] J. D. Edelstein, M. L. Trobo, F. A. Brito, and D. Bazeia, Kinks inside supersymmetric domain ribbons, Phys. Rev. D 57, 7561 (1998).

[29] M. Naganuma and M. Nitta, BPS domain walls in models with flat directions, Prog. Theor. Phys. 105, 501 (2001).

[30] M. Arai, S. Lee, and S. Shin, Walls in supersymmetric massive nonlinear sigma model on complex quadric surface, Phys. Rev. D 80, 125012 (2009).

[31] M. Arai and S. Shin, Walls of massive Káhler sigma models on $\mathrm{SO}(2 \mathrm{~N}) / \mathrm{U}(\mathrm{N})$ and $\mathrm{Sp}(\mathrm{N}) / \mathrm{U}(\mathrm{N})$, Phys. Rev. D 83, 125003 (2011).

[32] M. Nitta and S. Sasaki, BPS states in supersymmetric chiral models with higher derivative terms, Phys. Rev. D 90, 105001 (2014).

[33] S. B. Gudnason, M. Nitta, and S. Sasaki, BPS pion domain walls in the supersymmetric chiral Lagrangian, Phys. Rev. D 94, 025003 (2016).

[34] B. H. Lee, C. Park, and S. Shin, Vacua and walls of mass-deformed Kähler nonlinear sigma models on SO $(2 N) / U(N)$, Phys. Rev. D 96, 105017 (2017).

[35] M. Arai, A. Golubtsova, C. Park, and S. Shin, Vacua and walls of mass-deformed Kähler nonlinear sigma models on $S p(N) / U(N)$, Phys. Rev. D 97, 105012 (2018).

[36] E. R. C. Abraham and P. K. Townsend, $Q$ kinks, Phys. Lett. B 291, 85 (1992).

[37] E. R. C. Abraham and P. K. Townsend, More on $Q$ kinks: A $(1+1)$-dimensional analog of dyons, Phys. Lett. B 295, 225 (1992).

[38] J. P. Gauntlett, D. Tong, and P. K. Townsend, Supersymmetric intersecting domain walls in massive hyperKahler sigma models, Phys. Rev. D 63, 085001 (2001).

[39] J. P. Gauntlett, D. Tong, and P. K. Townsend, Multidomain walls in massive supersymmetric sigma models, Phys. Rev. D 64, 025010 (2001).

[40] D. Tong, The Moduli space of BPS domain walls, Phys. Rev. D 66, 025013 (2002).

[41] K. S. M. Lee, An Index theorem for domain walls in supersymmetric gauge theories, Phys. Rev. D 67, 045009 (2003).

[42] M. Arai, M. Naganuma, M. Nitta, and N. Sakai, Manifest supersymmetry for BPS walls in $N=2$ nonlinear sigma models, Nucl. Phys. B652, 35 (2003).

[43] M. Arai, M. Naganuma, M. Nitta, and N. Sakai, BPS wall in $N=2$ SUSY nonlinear sigma model with Eguchi-Hanson manifold, in A Garden of Quanta, edited by A. Arai et al. (World Scientific Publishing Co. Pte. Ltd., Singapore, 2003), pp. 299-325.

[44] A. Losev and M. Shifman, $N=2$ sigma model with twisted mass and superpotential: Central charges and solitons, Phys. Rev. D 68, 045006 (2003).

[45] Y. Isozumi, K. Ohashi, and N. Sakai, Exact wall solutions in five-dimensional SUSY QED at finite coupling, J. High Energy Phys. 11 (2003) 060. 
[46] M. Shifman and A. Yung, Localization of nonAbelian gauge fields on domain walls at weak coupling (D-brane prototypes II), Phys. Rev. D 70, 025013 (2004).

[47] Y. Isozumi, M. Nitta, K. Ohashi, and N. Sakai, Construction of Non-Abelian Walls and Their Complete Moduli Space, Phys. Rev. Lett. 93, 161601 (2004).

[48] Y. Isozumi, M. Nitta, K. Ohashi, and N. Sakai, Non-Abelian walls in supersymmetric gauge theories, Phys. Rev. D 70, 125014 (2004).

[49] M. Eto, Y. Isozumi, M. Nitta, K. Ohashi, K. Ohta, and N. Sakai, $D$-brane construction for non-Abelian walls, Phys. Rev. D 71, 125006 (2005).

[50] M. Eto, Y. Isozumi, M. Nitta, K. Ohashi, K. Ohta, N. Sakai, and Y. Tachikawa, Global structure of moduli space for BPS walls, Phys. Rev. D 71, 105009 (2005).

[51] M. Eto, M. Nitta, K. Ohashi, and D. Tong, Skyrmions from Instantons Inside Domain Walls, Phys. Rev. Lett. 95, 252003 (2005).

[52] M. Eto, T. Fujimori, Y. Isozumi, M. Nitta, K. Ohashi, K. Ohta, and N. Sakai, Non-Abelian vortices on cylinder: Duality between vortices and walls, Phys. Rev. D 73, 085008 (2006).

[53] M. Eto, Y. Isozumi, M. Nitta, K. Ohashi, and N. Sakai, Manifestly supersymmetric effective Lagrangians on BPS solitons, Phys. Rev. D 73, 125008 (2006).

[54] M. Eto, T. Fujimori, M. Nitta, K. Ohashi, and N. Sakai, Domain walls with non-Abelian clouds, Phys. Rev. D 77, 125008 (2008).

[55] Y. Isozumi, M. Nitta, K. Ohashi, and N. Sakai, All exact solutions of a 1/4 Bogomol'nyi-Prasad-Sommerfield equation, Phys. Rev. D 71, 065018 (2005).

[56] M. Eto, T. Fujimori, T. Nagashima, M. Nitta, K. Ohashi, and N. Sakai, Dynamics of strings between walls, Phys. Rev. D 79, 045015 (2009).

[57] D. Tong, TASI lectures on solitons: Instantons, monopoles, vortices and kinks, arXiv:hep-th/0509216.

[58] M. Eto, Y. Isozumi, M. Nitta, K. Ohashi, and N. Sakai, Solitons in the Higgs phase: The moduli matrix approach, J. Phys. A 39, R315 (2006).

[59] M. Shifman and A. Yung, Supersymmetric solitons and how they help us understand non-Abelian gauge theories, Rev. Mod. Phys. 79, 1139 (2007).

[60] M. Shifman and A. Yung, Supersymmetric Solitons (Cambridge University Press, Cambridge, United Kingdom, 2009).

[61] J. A. de Azcarraga, J. P. Gauntlett, J. M. Izquierdo, and P. K. Townsend, Topological Extensions of the Supersymmetry Algebra for Extended Objects, Phys. Rev. Lett. 63, 2443 (1989).

[62] G. W. Gibbons and P. K. Townsend, A Bogomolny Equation for Intersecting Domain Walls, Phys. Rev. Lett. 83, 1727 (1999).

[63] S. M. Carroll, S. Hellerman, and M. Trodden, Domain wall junctions are 1/4-BPS states, Phys. Rev. D 61, 065001 (2000).

[64] A. Gorsky and M. A. Shifman, More on the tensorial central charges in $N=1$ supersymmetric gauge theories (BPS wall junctions and strings), Phys. Rev. D 61, 085001 (2000).
[65] E. R. C. Abraham and P. K. Townsend, Intersecting extended objects in supersymmetric field theories, Nucl. Phys. B351, 313 (1991).

[66] G. Gabadadze and M. A. Shifman, D walls and junctions in supersymmetric gluodynamics in the large $\mathrm{N}$ limit suggest the existence of heavy hadrons, Phys. Rev. D 61, 075014 (2000).

[67] H. Oda, K. Ito, M. Naganuma, and N. Sakai, An exact solution of BPS domain wall junction, Phys. Lett. B 471, 140 (1999).

[68] M. A. Shifman and T. ter Veldhuis, Calculating the tension of domain wall junctions and vortices in generalized Wess-Zumino models, Phys. Rev. D 62, 065004 (2000).

[69] K. Ito, M. Naganuma, H. Oda, and N. Sakai, Nonnormalizable zero modes on BPS junctions, Nucl. Phys. B586, 231 (2000).

[70] D. Binosi and T. ter Veldhuis, Domain wall junctions in a generalized Wess-Zumino model, Phys. Lett. B 476, 124 (2000).

[71] S. Nam and K. Olsen, Domain wall junctions in supersymmetric field theories in $D=4$, J. High Energy Phys. 08 (2000) 001.

[72] S. M. Carroll, S. Hellerman, and M. Trodden, BPS domain wall junctions in infinitely large extra dimensions, Phys. Rev. D 62, 044049 (2000).

[73] M. Naganuma, M. Nitta, and N. Sakai, BPS walls and junctions in SUSY nonlinear sigma models, Phys. Rev. D 65, 045016 (2002).

[74] K. Kakimoto and N. Sakai, Domain wall junction in $N=2$ supersymmetric QED in four-dimensions, Phys. Rev. D 68, 065005 (2003).

[75] M. Eto, Y. Isozumi, M. Nitta, K. Ohashi, and N. Sakai, Webs of walls, Phys. Rev. D 72, 085004 (2005).

[76] M. Eto, Y. Isozumi, M. Nitta, K. Ohashi, and N. Sakai, Non-Abelian webs of walls, Phys. Lett. B 632, 384 (2006).

[77] M. Eto, Y. Isozumi, M. Nitta, K. Ohashi, K. Ohta, and N. Sakai, D-brane configurations for domain walls and their webs, AIP Conf. Proc. 805, 354 (2005).

[78] M. Eto, T. Fujimori, T. Nagashima, M. Nitta, K. Ohashi, and N. Sakai, Effective action of domain wall networks, Phys. Rev. D 75, 045010 (2007).

[79] M. Eto, T. Fujimori, T. Nagashima, M. Nitta, K. Ohashi, and N. Sakai, Dynamics of domain wall networks, Phys. Rev. D 76, 125025 (2007).

[80] T. Fujimori, M. Nitta, K. Ohta, N. Sakai, and M. Yamazaki, Intersecting solitons, amoeba and tropical geometry, Phys. Rev. D 78, 105004 (2008).

[81] S. Shin, Vacua, walls and junctions in $G_{N, N_{C}}$, Nucl. Phys. B946, 114701 (2019).

[82] S. Shin, Junctions of mass-deformed nonlinear sigma models on the Grassmann manifold, J. High Energy Phys. 111 (2019) 08.

[83] T. Kim and S. Shin, Junctions of mass-deformed nonlinear sigma models on $S O(2 N) / U(N)$ and $S p(N) / U(N)$ II, arXiv:2002.01923.

[84] P. M. Saffin, Tiling with Almost BPS Junctions, Phys. Rev. Lett. 83, 4249 (1999). 
[85] D. Bazeia and F. A. Brito, Bags, junctions, and networks of BPS and nonBPS defects, Phys. Rev. D 61, 105019 (2000).

[86] M. Eto, M. Kawaguchi, M. Nitta, and R. Sasaki, Exact solutions of domain wall junctions in arbitrary dimensions, arXiv:2001.07552.
[87] M. Nitta, Josephson vortices and the Atiyah-Manton construction, Phys. Rev. D 86, 125004 (2012).

[88] T. Fujimori, H. Iida, and M. Nitta, Field theoretical model of multilayered Josephson junction and dynamics of Josephson vortices, Phys. Rev. B 94, 104504 (2016). 